\title{
Contrasteric Stereochemical Dictation of the Cyclobutene Ring-Opening Reaction by a Vacant Boron p Orbital
}

\author{
Masahiro Murakami,* Ippei Usui, Munehiro Hasegawa, and Takanori Matsuda \\ Department of Synthetic Chemistry and Biological Chemistry, Kyoto University, \\ Katsura, Kyoto 615-8510, Japan
}

\section{Supporting Information}

General. All reactions were carried out under a nitrogen atmosphere. Column chromatography was performed with silica gel $60 \mathrm{~N}$ (Kanto) or Wakogel C-200 (Wako). ${ }^{1} \mathrm{H}$ and ${ }^{13} \mathrm{C}$ NMR spectra were recorded on a Varian Gemini $2000\left({ }^{1} \mathrm{H}\right.$ at $300.07 \mathrm{~Hz}$ and ${ }^{13} \mathrm{C}$ at $75.46 \mathrm{~Hz})$ spectrometer. ${ }^{11} \mathrm{~B}$ NMR spectra were recorded on a Varian Mercury $400\left({ }^{11} \mathrm{~B}\right.$ at $128.48 \mathrm{~Hz})$. Proton chemical shifts were referenced to residual solvent signals in $\mathrm{CDCl}_{3}(\delta$ 7.26), $\mathrm{C}_{6} \mathrm{D}_{6}(\delta 7.16)$, DMSO- $d_{6}(2.54)$, and toluene- $d_{8}(\delta 2.09)$. Carbon chemical shifts were referenced to the deuterated solvent signals in $\mathrm{CDCl}_{3}\left(\delta\right.$ 77.00), $\mathrm{C}_{6} \mathrm{D}_{6}(\delta$ 128.00), and DMSO- $d_{6}\left(\delta\right.$ 39.52). Boron chemical shifts were referenced to external standard $\mathrm{BF}_{3} \cdot \mathrm{OEt}_{2}$. High resolution mass spectra were recorded on a JEOL JMS-HX110A (FAB) or a JEOL JMS-SX102A (EI) spectrometer.

Materials. Unless otherwise noted, all chemicals and anhydrous solvents were obtained from commercial suppliers and used as received. Zinc-copper couple, ${ }^{1} 3$-bromo1-phenylcyclobutene, ${ }^{2}$ (dimethylphenylsilyl)pinacolborane ${ }^{3}$, and 1-bromobuta-1,3-diene ${ }^{4}$ were prepared according to the literature procedures. Trimethyl borate and triisopropyl borate were distilled prior to use. Diisopropylamine and $N, N, N^{\prime}, N^{\prime}-$ tetramethylethylenediamine were distilled from $\mathrm{CaH}_{2}$ and $\mathrm{KOH}$, respectively.

\footnotetext{
1) Danheiser, R. L.; Savariar, S.; Cha, D. D. Organic Synthese s; Wiley: New York, 1993; Coll. Vol. VIII, pp 82-86.

2) Murakami, M.; Hasegawa, M.; Igawa, H. J. Org. Chem. 2004, 69, 587.

3) Suginome, M.; Matsuda, T.; Ito, Y. Organometallics 2000, 19, 4647.

4) Keegstra, M. A.; Verkruijsse, H. D.; Andringa, H.; Brandsma, L. Synth. Commun. 1991, $21,721$.
} 


\section{Preparation of 3-Borylcyclobutenes 1 and 6}

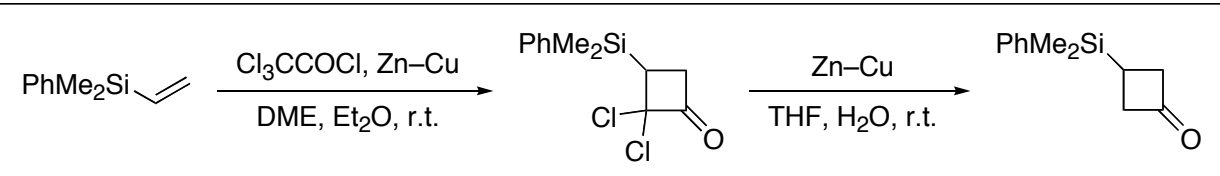

To a mixture of $\mathrm{Zn}-\mathrm{Cu}(16.6 \mathrm{~g}, 253 \mathrm{mmol})$ and dimethylphenylvinylsilane (13.8 g, $84.9 \mathrm{mmol})$ in $\mathrm{Et}_{2} \mathrm{O}(170 \mathrm{~mL})$ was added a solution of trichloroacetyl chloride $(18.7 \mathrm{~mL}$, $167 \mathrm{mmol})$ in 1,2-dimethoxyethane $(85 \mathrm{~mL})$ dropwise at room temperature. After being stirred for $36 \mathrm{~h}$ at room temperature, the mixture was filtered with Celite ${ }^{\circledR}\left(\mathrm{Et}_{2} \mathrm{O}\right)$, and the filtrate was washed successively with saturated $\mathrm{NaHCO}_{3}$ solution and brine. The organic layer was dried over $\mathrm{MgSO}_{4}$ and concentrated. The residue was purified by column chromatography on silica gel (hexane:AcOEt $=20: 1$ ) to give 2,2-dichloro-3(dimethylphenylsilyl)cyclobutanone $(12.6 \mathrm{~g}, 54 \%)$ as a yellow oil. To a mixture of $\mathrm{Zn}-\mathrm{Cu}$ (54.3 g, $830 \mathrm{mmol})$ and 2,2-dichloro-3-(dimethylphenylsilyl)cyclobutanone (6.3 g, 23 $\mathrm{mmol})$ in THF $(120 \mathrm{~mL})$ was added water $(12 \mathrm{~mL})$ dropwise. After being stirred for $1 \mathrm{~h}$ at room temperature, the mixture was filtered with Celite ${ }^{\circledR}\left(\mathrm{Et}_{2} \mathrm{O}\right)$, and the filtrate was washed with brine (three times) and dried over $\mathrm{MgSO}_{4}$. Evaporation of the solvents afforded almost pure 3-(dimethylphenylsilyl)cyclobutanone (quant.).

3-(Dimethylphenylsilyl)cyclobutanone. ${ }^{1} \mathrm{H} \mathrm{NMR}\left(\mathrm{CDCl}_{3}\right) \delta 0.34(\mathrm{~s}, 6 \mathrm{H}), 1.81$ (tt, $J=10.8$, 8.4 Hz, 1H), 2.87-2.98 (m, 2H), 3.15-3.26 (m, 2H), 7.36-7.41 (m, 3H), 7.49-7.53 (m, 2H); ${ }^{13} \mathrm{C} \mathrm{NMR}\left(\mathrm{CDCl}_{3}\right) \delta-5.0,8.8,49.0,128.0,129.4,133.7,136.8,207.5$; HRMS (FAB) calcd for $\mathrm{C}_{12} \mathrm{H}_{17} \mathrm{OSi}\left(\mathrm{M}^{+}+\mathrm{H}\right)$ 205.1049, found 205.1049.

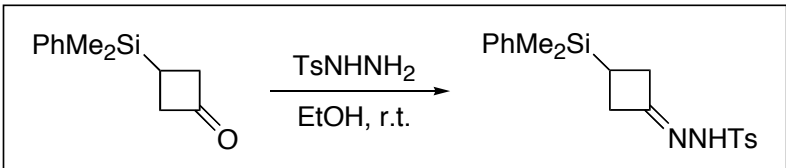

A solution of 3-(dimethylphenylsilyl)cyclobutanone (2.28 g, $11.2 \mathrm{mmol})$ in ethanol (14 $\mathrm{mL})$ was added dropwise to a suspension of $p$-tosylhydrazine $(2.27 \mathrm{~g}, 12.3 \mathrm{mmol})$ in ethanol $(30 \mathrm{~mL})$ at room temperature. Within 30-60 min the tosylhydrazone began to precipitate. In the meantime, the mixture was sonicated to facilitate dispersion. The white 
solid was filtered, washed with ethanol then with $\mathrm{Et}_{2} \mathrm{O}$, and dried in vacuo to afford 3(dimethylphenylsilyl)cyclobutanone tosylhydrazone $(3.56 \mathrm{~g}, 86 \%)$.

3-(Dimethylphenylsilyl)cyclobutanone Tosylhydrazone. ${ }^{1} \mathrm{H}$ NMR (DMSO- $\left.d_{6}\right) \delta 0.21$ (s, $3 \mathrm{H}), 0.22(\mathrm{~s}, 3 \mathrm{H}), 1.83(\mathrm{tt}, J=10.9,7.1 \mathrm{~Hz}, 1 \mathrm{H}), 2.42(\mathrm{~s}, 3 \mathrm{H}), 2.57-2.77(\mathrm{~m}, 2 \mathrm{H}), 2.91-3.05$ $(\mathrm{m}, 2 \mathrm{H}), 7.34-7.45(\mathrm{~m}, 5 \mathrm{H}), 7.48-7.53(\mathrm{~m}, 2 \mathrm{H}), 7.69-7.75(\mathrm{~m}, 2 \mathrm{H}), 10.2(\mathrm{~s}, 1 \mathrm{H}) ;{ }^{13} \mathrm{C} \mathrm{NMR}$ $\left(\mathrm{DMSO}_{6}\right) \delta-5.6,-5.3,11.6,21.0,34.1,34.6,127.3,127.8,129.2,129.4,133.5,136.3$, 137.1, 143.0, 159.4; HRMS (EI) calcd for $\mathrm{C}_{19} \mathrm{H}_{25} \mathrm{~N}_{2} \mathrm{O}_{2} \mathrm{SSi}\left(\mathrm{M}^{+}+\mathrm{H}\right)$ 373.1406, found 373.1404

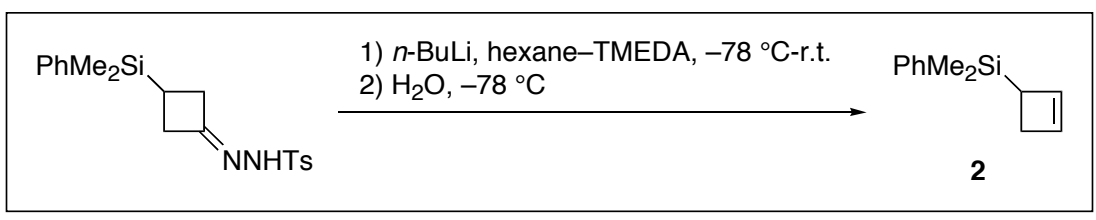

To a mixture of the hydrazone $(22.6 \mathrm{~g}, 60.8 \mathrm{mmol})$ and $N, N, N^{\prime}, N^{\prime}-$ tetramethylethylenediamine $(23 \mathrm{~mL})$ in hexane $(226 \mathrm{~mL})$ was added $n$-BuLi $(1.67 \mathrm{M}$ in hexane, $80 \mathrm{~mL}, 134 \mathrm{mmol}$ ) dropwise at $-78{ }^{\circ} \mathrm{C}$, and then the mixture was allowed to warm gradually to room temperature. After being stirred for $15 \mathrm{~h}$, the mixture was quenched with water at $-78{ }^{\circ} \mathrm{C}$, added $1 \mathrm{~N} \mathrm{HCl}(150 \mathrm{~mL})$, and extracted with $\mathrm{Et}_{2} \mathrm{O}$. The organic layer was washed with saturated $\mathrm{NaHCO}_{3}$ aqueous solution, dried over $\mathrm{MgSO}_{4}$, and concentrated. The residue was purified by column chromatography on silica gel (hexane) to afford 2 $(6.97 \mathrm{~g}, 61 \%)$.

3-(Dimethylphenylsilyl)cyclobutene (2). ${ }^{1} \mathrm{H}$ NMR $\left(\mathrm{CDCl}_{3}\right) \delta 0.28(\mathrm{~s}, 3 \mathrm{H}), 0.30$ (s, 3H), 2.44 (d with unresolved coupling, $J=14.0 \mathrm{~Hz}, 1 \mathrm{H}$ ), 2.66 (d with unresolved coupling, $J=$ $4.4 \mathrm{~Hz}, 1 \mathrm{H}), 2.76(\mathrm{dd}, J=14.0,4.4 \mathrm{~Hz}, 1 \mathrm{H}), 6.02(\mathrm{dt}, J=2.5,2.4 \mathrm{~Hz}, 1 \mathrm{H}), 6.13$ (d, $J=2.5$ $\mathrm{Hz}, 1 \mathrm{H}), 7.37-7.41(\mathrm{~m}, 3 \mathrm{H}), 7.53-7.58(\mathrm{~m}, 2 \mathrm{H}) ;{ }^{13} \mathrm{C} \mathrm{NMR}\left(\mathrm{CDCl}_{3}\right) \delta-5.0,-4.9,32.5,33.9$, 127.6, 128.9, 133.6, 134.3, 138.5, 138.8; HRMS (EI) calcd for $\mathrm{C}_{12} \mathrm{H}_{16} \mathrm{Si}$ 188.1021, found 188.1025 . 


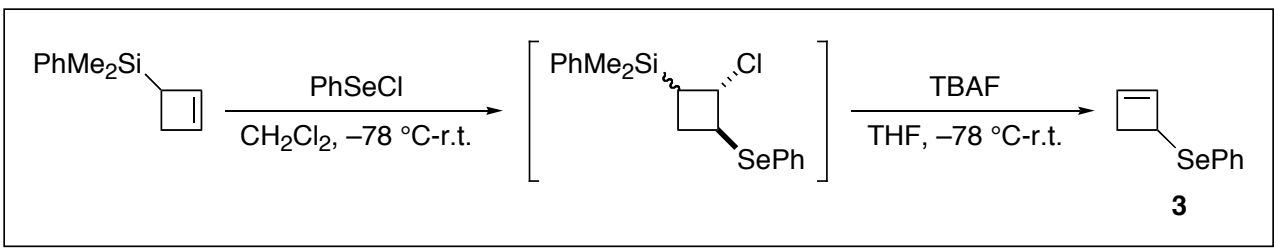

To a solution of $\mathrm{PhSeCl}(1.61 \mathrm{~g}, 8.4 \mathrm{mmol})$ in $\mathrm{CH}_{2} \mathrm{Cl}_{2}(23 \mathrm{~mL})$ was added 2 (1.59 g, $8.4 \mathrm{mmol}$ ) dropwise at $-78{ }^{\circ} \mathrm{C}$, and the mixture was gradually warmed to room temperature. After the mixture was stirred for $3 \mathrm{~h}$, the solvent was removed. The residue was cooled to $-78{ }^{\circ} \mathrm{C}$ again, and dissolved in THF $(7 \mathrm{~mL})$. To the solution was added TBAF (1.0 $\mathrm{M}$ in THF, $3.0 \mathrm{~mL}, 3.0 \mathrm{mmol}$ ), and the mixture was stirred for $5 \mathrm{~h}$ (at $-78{ }^{\circ} \mathrm{C}$ to r.t.). After evaporation of the solvent, the residue was passed through a plug of Florisil ${ }^{\circledR}\left(\mathrm{Et}_{2} \mathrm{O}\right)$. Column chromatography on silica gel (hexane) afforded $3(0.79 \mathrm{~g}, 45 \%)$.

3-Phenylselenylcyclobutene (3). ${ }^{1} \mathrm{H}$ NMR $\left(\mathrm{C}_{6} \mathrm{D}_{6}\right) \delta 2.50(\mathrm{~d}, J=14.7 \mathrm{~Hz}, 1 \mathrm{H}), 2.77(\mathrm{dd}, J=$ 14.7, 4.6 Hz, 1H), 4.25 (d, $J=4.6 \mathrm{~Hz}, 1 \mathrm{H}), 5.69$ (d, $J=2.3 \mathrm{~Hz}, 1 \mathrm{H}), 5.87$ (d, $J=2.3 \mathrm{~Hz}$, $1 \mathrm{H})$, 6.86-6.91 (m, 1H), 6.96-7.01 (m, 2H), 7.44-7.53 (m, 2H); HRMS (EI) calcd for $\mathrm{C}_{10} \mathrm{H}_{10} \mathrm{Se} 209.9948$, found 209.9953.

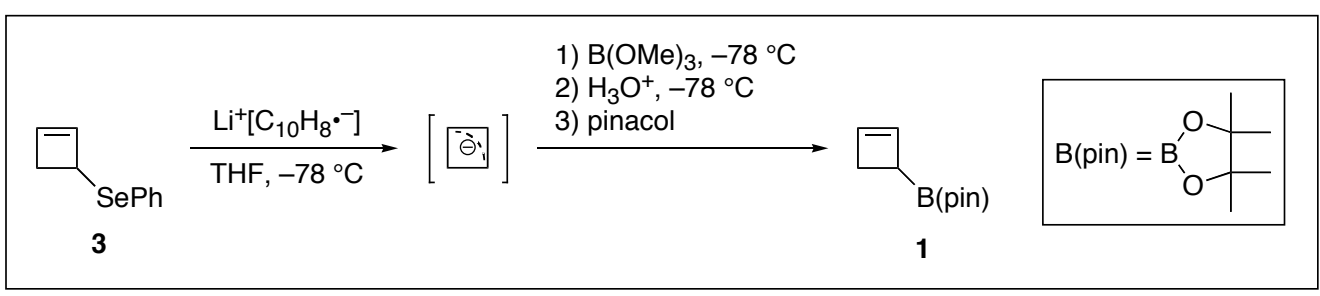

A mixture of naphthalene $(1.03 \mathrm{~g}, 8.0 \mathrm{mmol})$ and lithium $(57.3 \mathrm{mg}, 8.3 \mathrm{mmol})$ in THF $(20 \mathrm{~mL})$ was stirred for $3 \mathrm{~h}$ at room temperature to give black lithium naphthalenide solution. To the solution was added a solution of $\mathbf{3}(788 \mathrm{mg}, 3.77 \mathrm{mmol})$ in THF (4 mL) at $-78^{\circ} \mathrm{C}$, and the mixture was stirred for $3 \mathrm{~h}$. To the mixture was added $\mathrm{B}(\mathrm{OMe})_{3}(0.87 \mathrm{~mL}$, $7.4 \mathrm{mmol})$ at $-78^{\circ} \mathrm{C}$, and the reaction was quenched with $1 \mathrm{~N} \mathrm{HCl}$ solution $(15 \mathrm{~mL})$. The aqueous layer was extracted with ether containing pinacol (886 mg, $7.5 \mathrm{mmol}$ ), then ether (four times). The combined organic extracts were dried over $\mathrm{MgSO}_{4}$, and concentrated (100 $\mathrm{mmHg}$ ). The residue was purified column chromatography on silica gel (pentane:ether = 
$50: 1,25: 1$, then $12: 1)$ to afford $1(220 \mathrm{mg}, 32 \%)$.

3-(4,4,5,5-Tetramethyl-1,3,2-dioxaborolan-2-yl)cyclobutene (1). ${ }^{1} \mathrm{H}$ NMR $\left(\mathrm{C}_{6} \mathrm{D}_{6}\right) \delta 1.04$ (s, 12H), 2.71 (dd with unresolved coupling, $J=12.8,4.8 \mathrm{~Hz}, 1 \mathrm{H}), 2.76(\mathrm{~d}, J=4.8 \mathrm{~Hz}, 1 \mathrm{H})$, 2.85 (d with unresolved coupling, $J=12.8 \mathrm{~Hz}, 1 \mathrm{H}$ ), 6.02 (d with unresolved coupling, $J=$ $2.9 \mathrm{~Hz}, 1 \mathrm{H}), 6.21(\mathrm{~d}, J=2.9 \mathrm{~Hz}, 1 \mathrm{H}) ;{ }^{13} \mathrm{C} \mathrm{NMR}\left(\mathrm{C}_{6} \mathrm{D}_{6}\right) \delta 24.9,33.4,83.0,136.1,138.2$ [carbon attached to boron was not observed due to quadrupole broadening caused by the boron nucleus]; ${ }^{11} \mathrm{~B}$ NMR $\left(\mathrm{C}_{6} \mathrm{D}_{6}\right) \delta 33.3$; HRMS (EI) calcd for $\mathrm{C}_{10} \mathrm{H}_{17} \mathrm{BO}_{2}$ 180.1322, found 180.1322.

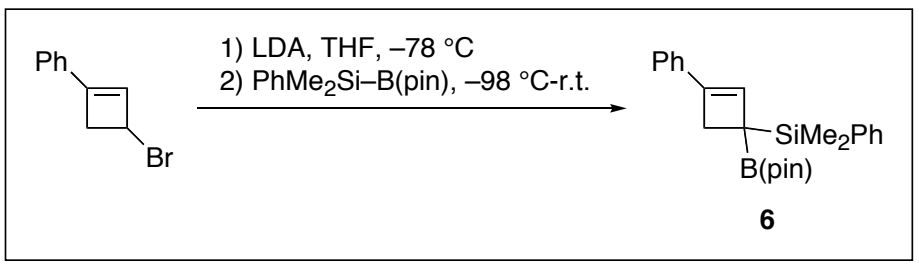

A solution of LDA $(2.50 \mathrm{mmol})$ in THF $(6.5 \mathrm{~mL})$ was added to a solution of 3-bromo1-phenylcyclobutene (525 mg, $2.51 \mathrm{mmol}$ ) and (dimethylphenylsilyl)pinacolborane (721 $\mathrm{mg}, 2.75 \mathrm{mmol})$ in $\mathrm{THF}(13 \mathrm{~mL})$ at $-98{ }^{\circ} \mathrm{C}$. The reaction mixture was stirred for $10 \mathrm{~min}$ at $-98{ }^{\circ} \mathrm{C}$, and then allowed to gradually warm to room temperature. The reaction was quenched with saturated $\mathrm{NH}_{4} \mathrm{Cl}$ aqueous solution, extracted with $\mathrm{Et}_{2} \mathrm{O}$, dried over $\mathrm{MgSO}_{4}$, and concentrated. Column chromatography on silica gel (hexane:AcOEt $=20: 1$ ) afforded 6 (574 mg, 59\%).

\section{3-(Dimethylphenylsilyl)-1-phenyl-3-(4,4,5,5-Tetramethyl-1,3,2-dioxaborolan-2-}

yl)cyclobutene (6). ${ }^{1} \mathrm{H}$ NMR $\left(\mathrm{CDCl}_{3}\right) \delta 0.32$ (s, 3H), 0.35 (s, 3H), 1.11 (s, 12H), 2.83 (d, $J$ $=13.7 \mathrm{~Hz}, 1 \mathrm{H}), 3.08(\mathrm{~d}, J=13.7 \mathrm{~Hz}, 1 \mathrm{H}), 6.52(\mathrm{~s}, 1 \mathrm{H}), 7.14-7.21(\mathrm{~m}, 2 \mathrm{H}), 7.23-7.29(\mathrm{~m}$, 3H), 7.29-7.34 (m, 3H), 7.54-7.62 (m, 2H); ${ }^{13} \mathrm{C} \mathrm{NMR}\left(\mathrm{CDCl}_{3}\right) \delta-4.5,-4.4,24.7,24.9,33.6$, 83.0, 123.5, 126.6, 127.4, 128.2, 128.4, 131.2, 134.0, 135.0, 138.3, 142.4; HRMS (FAB) calcd for $\mathrm{C}_{24} \mathrm{H}_{31} \mathrm{BO}_{2} \mathrm{Si} 390.2186$, found 390.2193. 


\section{Kinetic Studies on Ring-Opening Reactions of 3-Borylcyclobutenes 1 and 6}

Rate Measurements: The ring-opening reactions of $\mathbf{1}$ and $\mathbf{6}$ were monitored using ${ }^{1} \mathrm{H}$ NMR spectroscopy. The boryl-substituted cyclobutene was dissolved in toluene- $d_{8}$. The solution in an NMR tube was heated in a temperature-controlled oil bath at the specified temperature. The reaction was intercepted at intervals, and the ${ }^{1} \mathrm{H}$ NMR spectrum was recorded. The conversion was determined on the basis of the ${ }^{1} \mathrm{H}$ NMR integrations of the vinylic protons of the reactants and products. The \%conversion versus time data were subjected to least-squares analysis.

\section{Ring-Opening Reaction of 1}

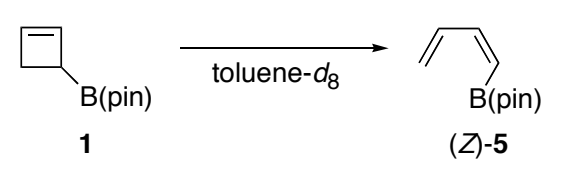

\begin{tabular}{cc|cc|cc}
\hline \multicolumn{2}{c|}{$70^{\circ} \mathrm{C}$} & \multicolumn{2}{|c|}{$81{ }^{\circ} \mathrm{C}$} & \multicolumn{2}{c}{$9{ }^{\circ} \mathrm{C}$} \\
\hline time/h & conv. $/ \%$ & time/h & conv. $/ \%$ & time/h & conv. $/ \%$ \\
\hline 0 & 0 & 0 & 0 & 0 & 0 \\
2 & 22 & 0.5 & 18 & 0.5 & 41 \\
3 & 31 & 1 & 31 & 1 & 63 \\
4 & 40 & 2 & 52 & 1.5 & 79 \\
6 & 51 & 3 & 67 & 2 & 87 \\
8 & 62 & & & & \\
11 & 73 & & & & \\
25 & 95 & & & & \\
\hline
\end{tabular}

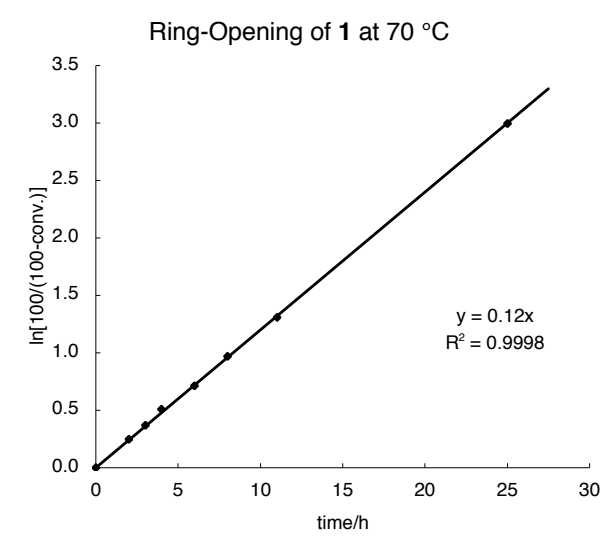



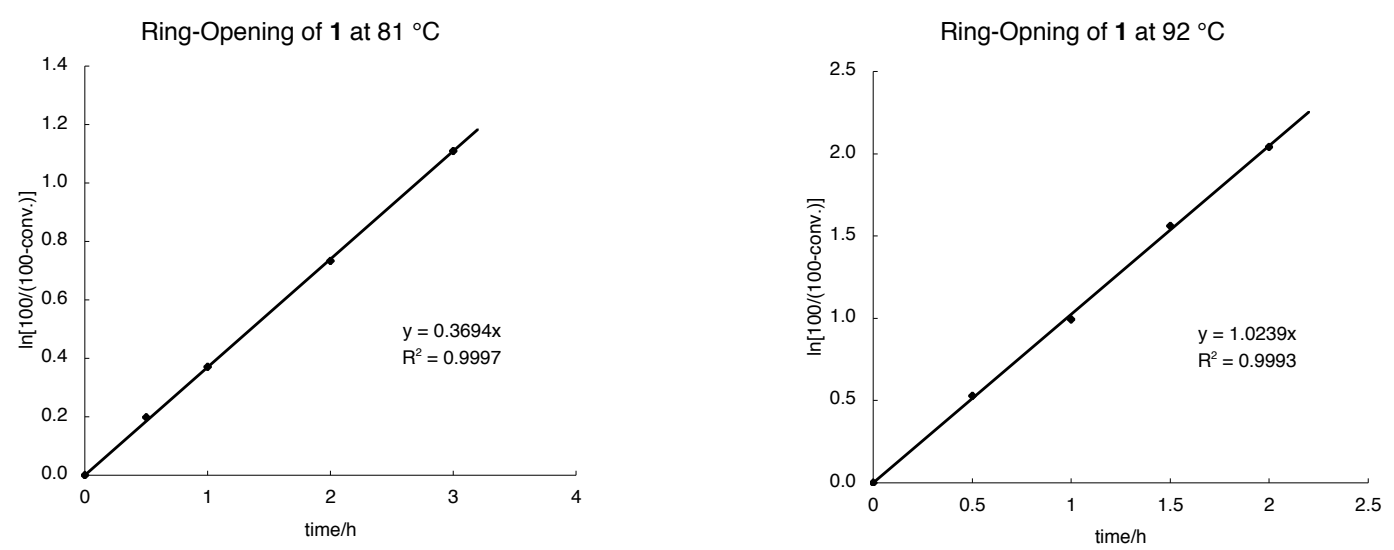

\begin{tabular}{clcc}
\hline$T\left({ }^{\circ} \mathrm{C}\right)$ & $k\left(\mathrm{~h}^{-1}\right)$ & $1000 / T\left(\mathrm{~K}^{-1}\right)$ & $\ln k$ \\
\hline 70 & 0.12 & 2.91 & -2.12 \\
81 & 0.3694 & 2.82 & -1.00 \\
92 & 1.0239 & 2.74 & 0.02 \\
\hline
\end{tabular}

$$
\begin{aligned}
& \ln A=33.5 ; A=10^{14.5} \mathrm{~h}^{-1} \\
& E_{\mathrm{a}}=1000 R \times 12.2=24.3 \mathrm{kcal} / \mathrm{mol} \\
& k=10^{14.5} \exp (-24.3 / R T) \mathrm{h}^{-1}
\end{aligned}
$$

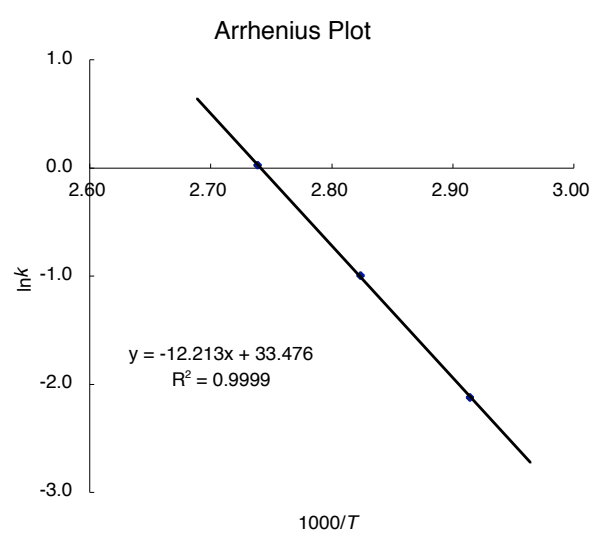

(Z)-1-(4,4,5,5-Tetramethyl-1,3,2-dioxaborolan-2-yl)buta-1,3-diene ((Z)-5). ${ }^{1} \mathrm{H} \quad \mathrm{NMR}$ (toluene- $d_{8}$ ) $\delta 1.04$ (s, 12H), 5.169 (d with unresolved coupling, $J=11.1 \mathrm{~Hz}$ ), 5.174 (d with unresolved coupling, $J=15.3 \mathrm{~Hz}), 5.65(\mathrm{dq}, J=13.5,0.9 \mathrm{~Hz}), 6.81(\mathrm{t}, J=11.9 \mathrm{~Hz}), 7.56$ (dddd, $J=17.6,10.9,9.4,1.0 \mathrm{~Hz}) ;{ }^{13} \mathrm{C}$ NMR $\left(\mathrm{C}_{6} \mathrm{D}_{6}\right) \delta 24.9,83.0,121.2,137.9,151.4$; HRMS (FAB) calcd for $\mathrm{C}_{10} \mathrm{H}_{17} \mathrm{BO}_{2} 180.1322$, found 180.1321 .

The stereoisomer, $(E)-\mathbf{5}$, was synthesized independently by reaction of triisopropyl borate with buta-1,3-dien-1-yllithium, which was generated in situ from (E)-1-bromobuta-1,3diene and $t$-BuLi. $(\boldsymbol{E})-5:{ }^{1} \mathrm{H}$ NMR (toluene- $\left.d_{8}\right) \delta 1.08(\mathrm{~s}, 12 \mathrm{H}$ ), 4.98 (d with unresolved coupling, $J=10.2 \mathrm{~Hz}, 1 \mathrm{H}$ ), 5.12 (d with unresolved coupling, $J=17.1 \mathrm{~Hz}, 1 \mathrm{H}), 5.74(\mathrm{~d}, J=$ $17.7 \mathrm{~Hz}, 1 \mathrm{H}), 6.28(\mathrm{ddt}, J=17.1,0.8,10.2 \mathrm{~Hz}, 1 \mathrm{H}), 7.23(\mathrm{dd}, J=17.7,10.8 \mathrm{~Hz}, 1 \mathrm{H}) ;{ }^{13} \mathrm{C}$ 
$\operatorname{NMR}\left(\mathrm{C}_{6} \mathrm{D}_{6}\right) \delta 24.9,83.1,120.7,139.3,150.8$; HRMS (FAB) calcd for $\mathrm{C}_{10} \mathrm{H}_{17} \mathrm{BO}_{2}$ 180.1322, found 180.1321 .

\section{Ring-Opening Reaction of 6}

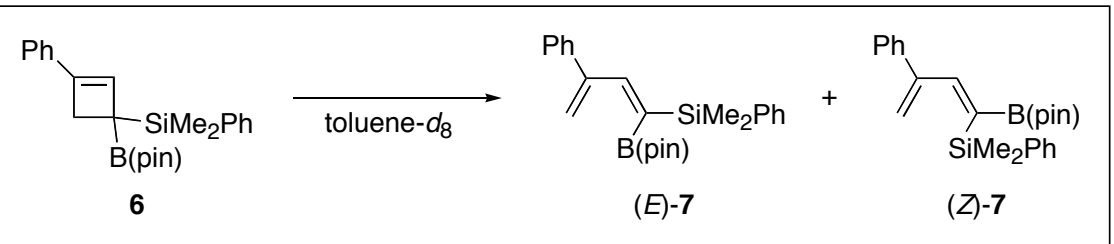

\begin{tabular}{cc|cc|cc}
\hline \multicolumn{2}{c|}{$80{ }^{\circ} \mathrm{C}$} & \multicolumn{2}{|c|}{$90{ }^{\circ} \mathrm{C}$} & \multicolumn{2}{c}{$100{ }^{\circ} \mathrm{C}$} \\
\hline time/h & conv. $/ \%$ & time/h & conv./\% & time/h & conv. $/ \%$ \\
\hline 0 & 0 & 0 & 0 & 0 & 0 \\
0.5 & 9 & 0.5 & 26 & 0.5 & 48 \\
1 & 16 & 1 & 44 & 1 & 65 \\
2 & 27 & & & & \\
\hline
\end{tabular}
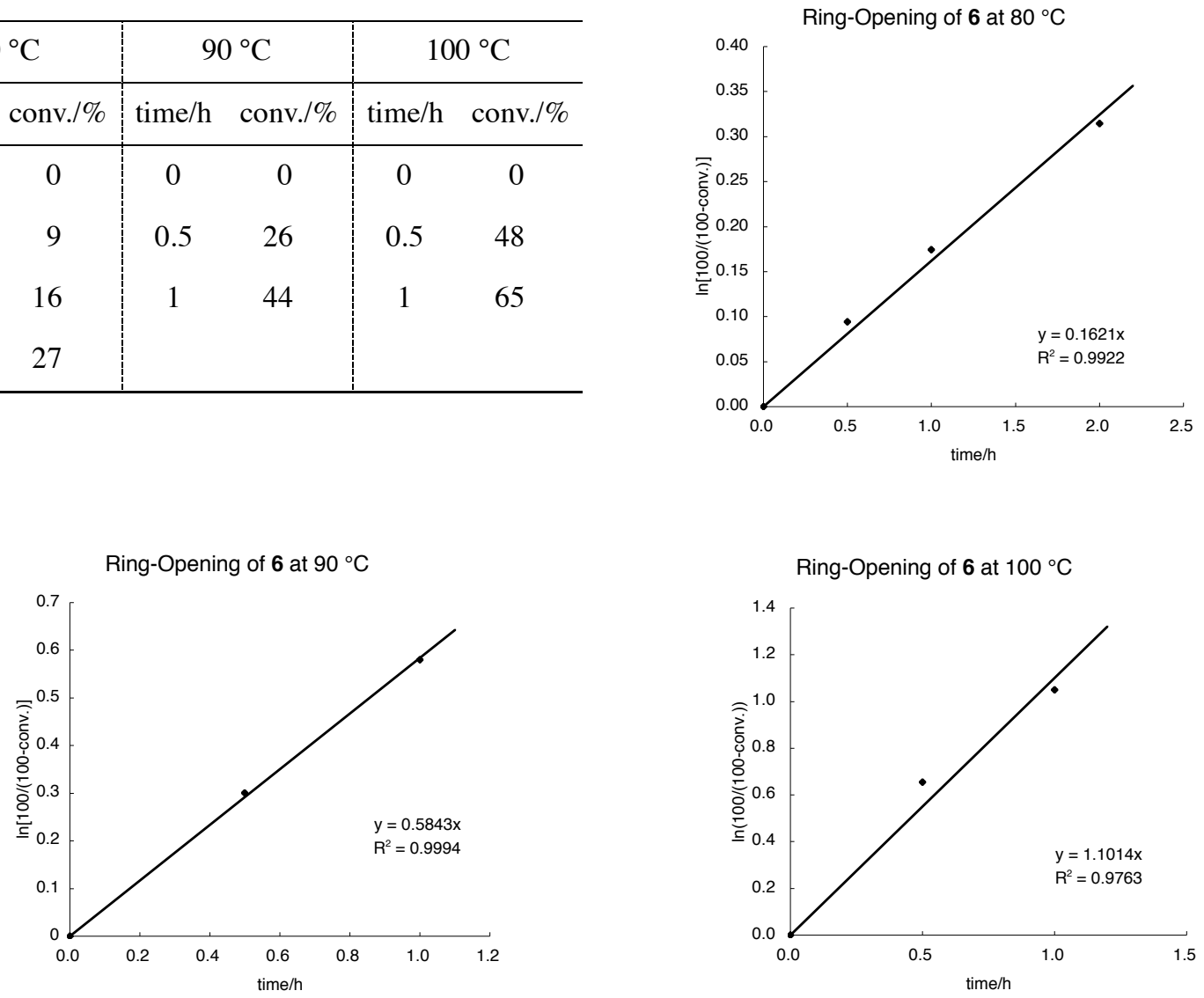


\begin{tabular}{cccc}
\hline$T\left({ }^{\circ} \mathrm{C}\right)$ & $k\left(\mathrm{~h}^{-1}\right)$ & $1000 / T\left(\mathrm{~K}^{-1}\right)$ & $\ln k$ \\
\hline 80 & 0.1621 & 2.83 & -1.82 \\
90 & 0.5843 & 2.75 & -0.54 \\
100 & 1.1014 & 2.68 & 0.10 \\
\hline
\end{tabular}

$$
\begin{aligned}
& \ln A=34.1 ; A=10^{14.8} \mathrm{~h}^{-1} \\
& E_{\mathrm{a}}=1000 R \times 12.7=25.1 \mathrm{kcal} / \mathrm{mol} \\
& k=10^{14.8} \exp (-25.1 / R T) \mathrm{h}^{-1}
\end{aligned}
$$

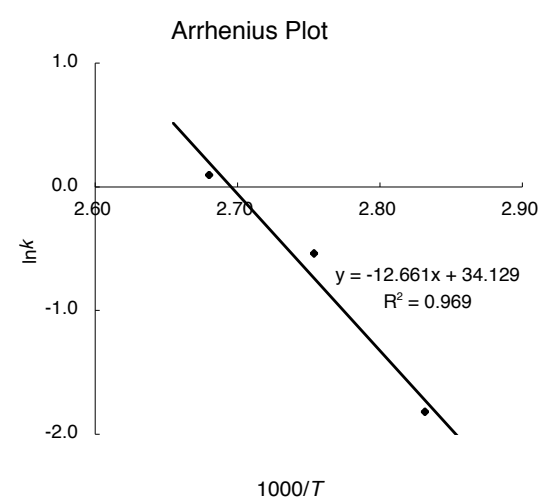

The two stereoisomers (E)-7 and (Z)-7 were separated by HPLC. (E)-1(Dimethylphenylsilyl)-3-phenyl-1-(4,4,5,5-tetramethyl-1,3,2-dioxaborolan-2-yl)buta1,3-diene ((E)-7). ${ }^{1} \mathrm{H}$ NMR $\left(\mathrm{CDCl}_{3}\right) \delta 0.45(\mathrm{~s}, 6 \mathrm{H}), 1.00(\mathrm{~s}, 12 \mathrm{H}), 5.34(\mathrm{~d}, J=1.5 \mathrm{~Hz}, 1 \mathrm{H})$, $5.36(\mathrm{~d}, J=1.2 \mathrm{~Hz}, 1 \mathrm{H}), 7.01(\mathrm{~s}, 1 \mathrm{H}), 7.26-7.38(\mathrm{~m}, 8 \mathrm{H}), 7.57-7.61(\mathrm{~m}, 2 \mathrm{H}) ;{ }^{13} \mathrm{C} \mathrm{NMR}$ $\left(\mathrm{CDCl}_{3}\right) \delta-2.0,24.8,83.1,115.5,127.2,127.6,128.2,128.9,134.3,138.4,140.2,149.5$, 152.2; HRMS (FAB) calcd for $\mathrm{C}_{24} \mathrm{H}_{31} \mathrm{BO}_{2} \mathrm{Si} 390.2186$, found 390.2189.

The stereochemical assignment of $(E)-7$ was established by ${ }^{1} \mathrm{H}$ NOE experiments as shown below.

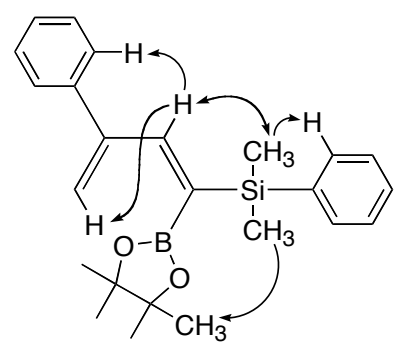

$(E)-7$

(Z)-1-(Dimethylphenylsilyl)-3-phenyl-1-(4,4,5,5-tetramethyl-1,3,2-dioxaborolan-2yl)buta-1,3-diene ((Z)-7). ${ }^{1} \mathrm{H}$ NMR $\left(\mathrm{CDCl}_{3}\right) \delta 0.38(\mathrm{~s}, 6 \mathrm{H}), 1.20(\mathrm{~s}, 12 \mathrm{H}), 5.05(\mathrm{t}, J=1.5$ $\mathrm{Hz}, 1 \mathrm{H}), 5.34(\mathrm{~d}, J=1.5 \mathrm{~Hz}, 1 \mathrm{H}), 7.25-7.38(\mathrm{~m}, 8 \mathrm{H}), 7.52-7.56(\mathrm{~m}, 2 \mathrm{H}), 7.69(\mathrm{~s}, 1 \mathrm{H}) ;{ }^{13} \mathrm{C}$ NMR $\left(\mathrm{CDCl}_{3}\right) \delta-0.1,25.0,83.4,115.0,126.8,127.6,127.9,128.4,128.5,134.1,139.1$, 
141.2, 148.6, 158.2; HRMS (FAB) calcd for $\mathrm{C}_{24} \mathrm{H}_{31} \mathrm{BO}_{2} \mathrm{Si} 390.2186$, found 390.2182. 


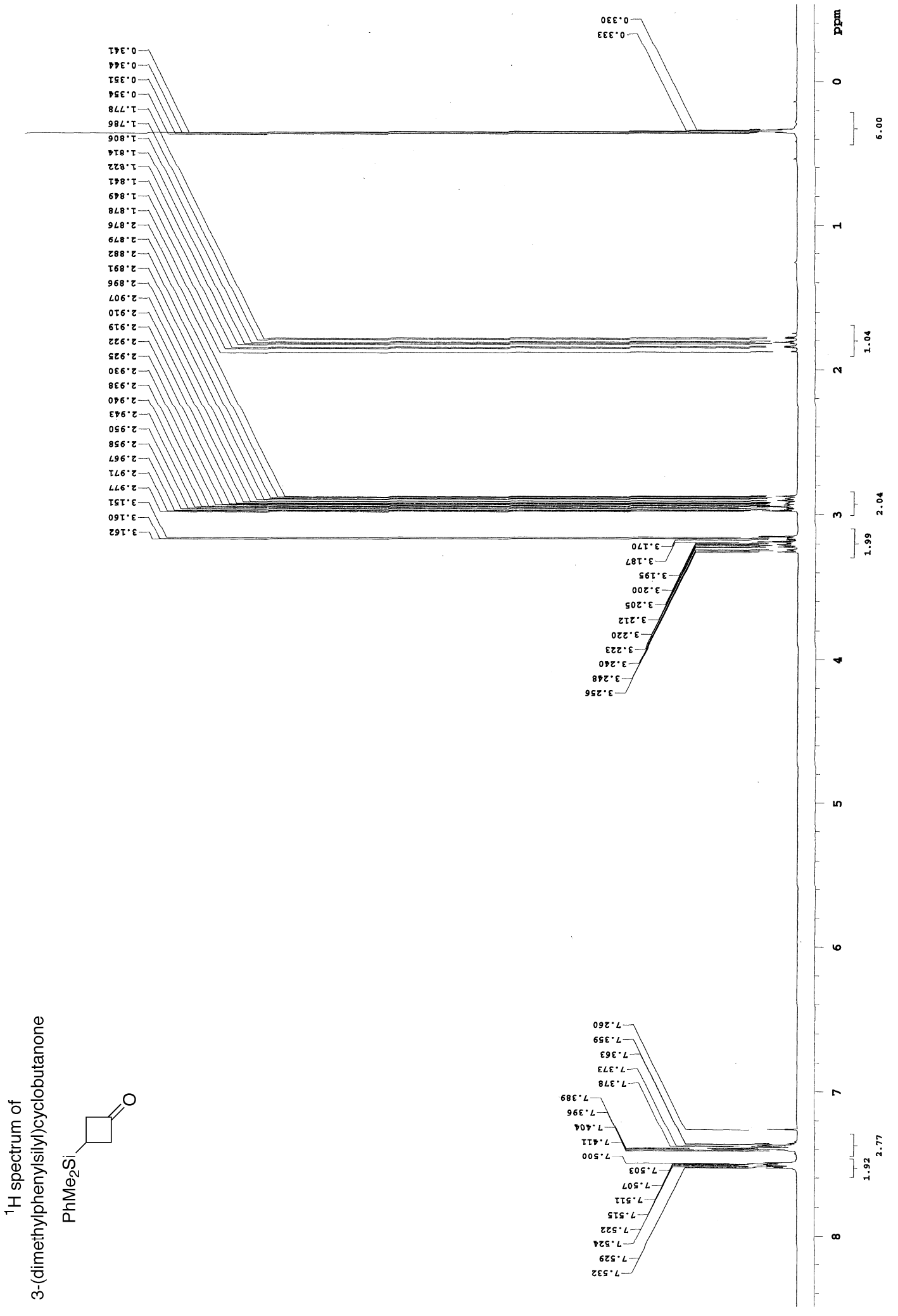




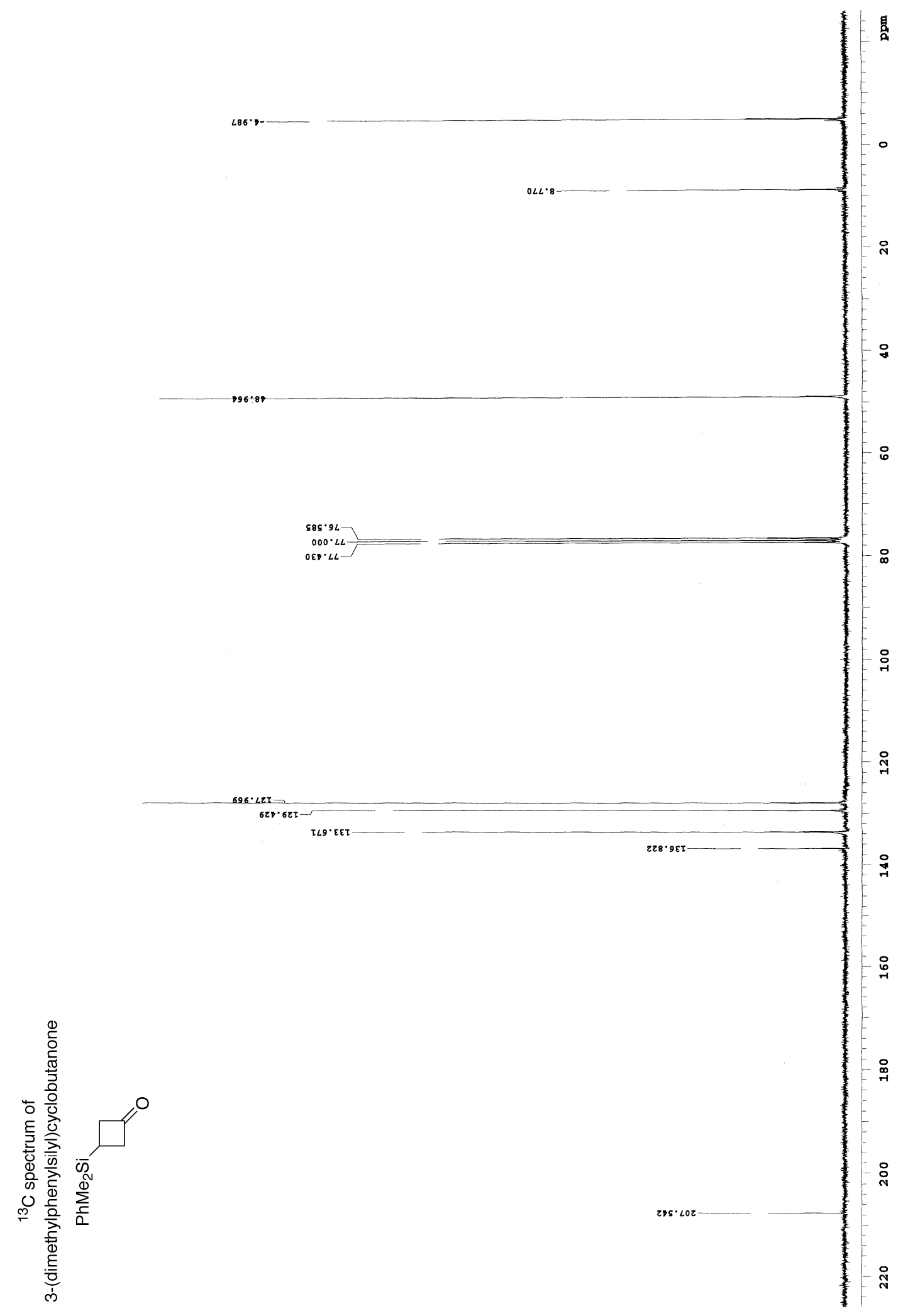




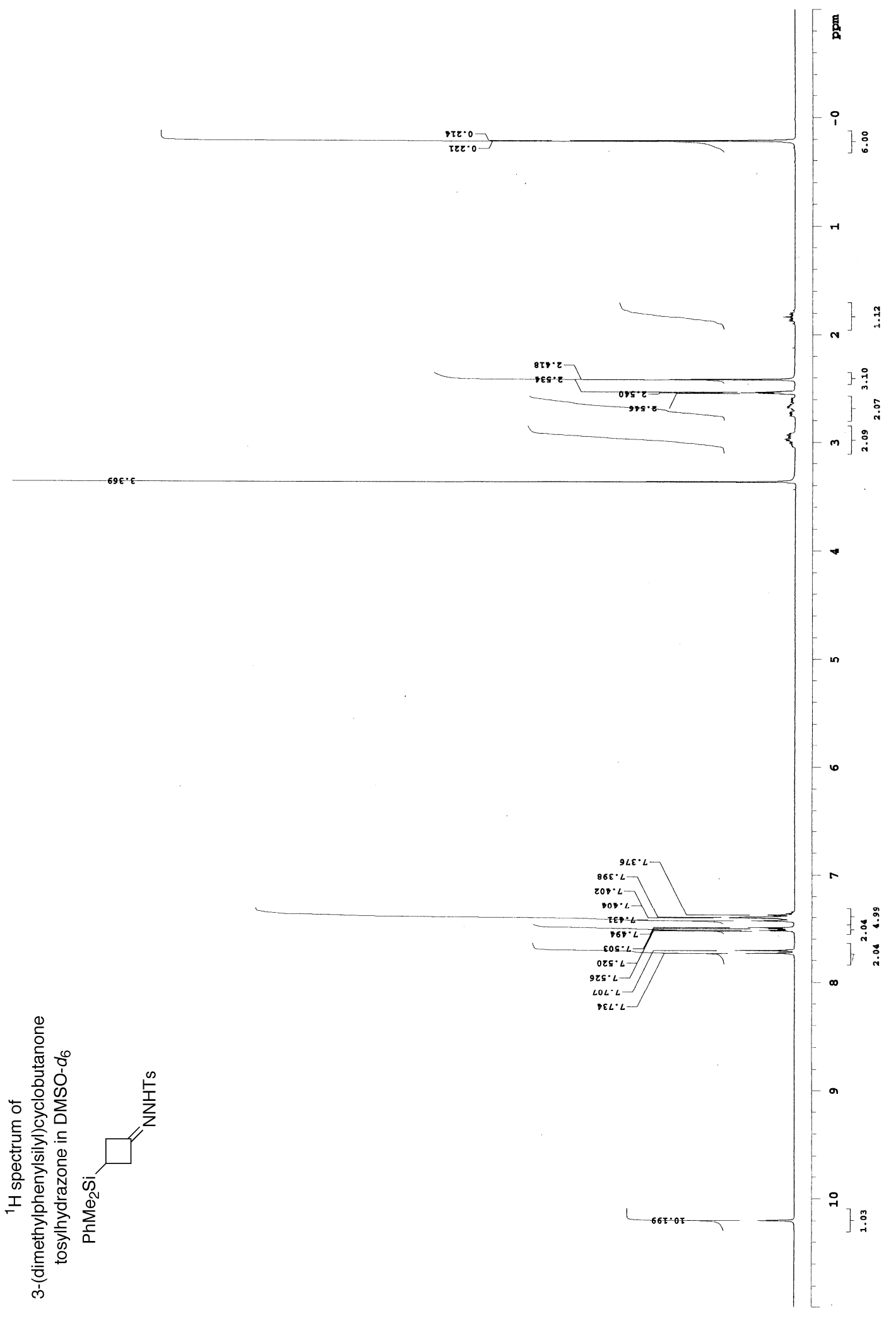




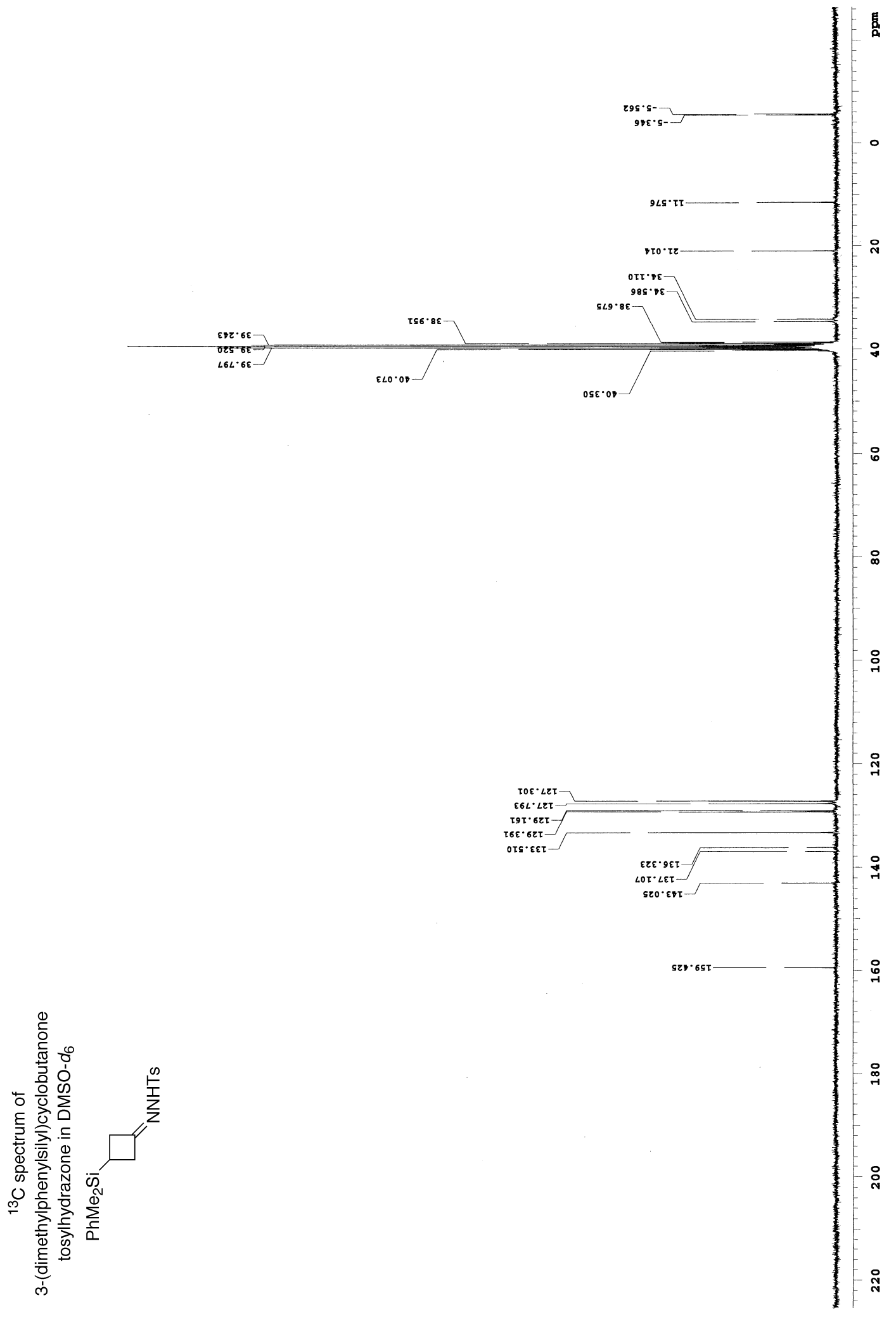




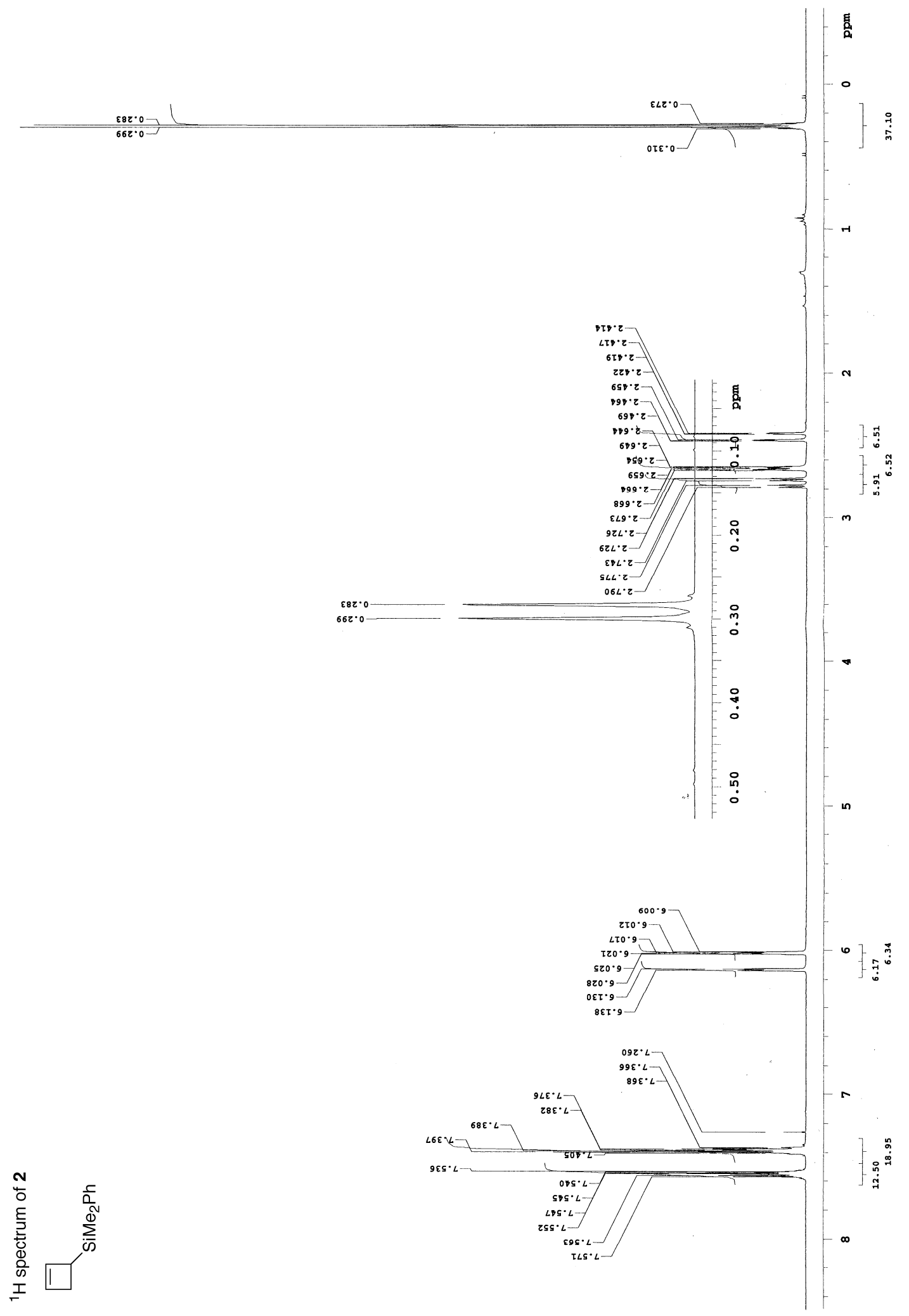




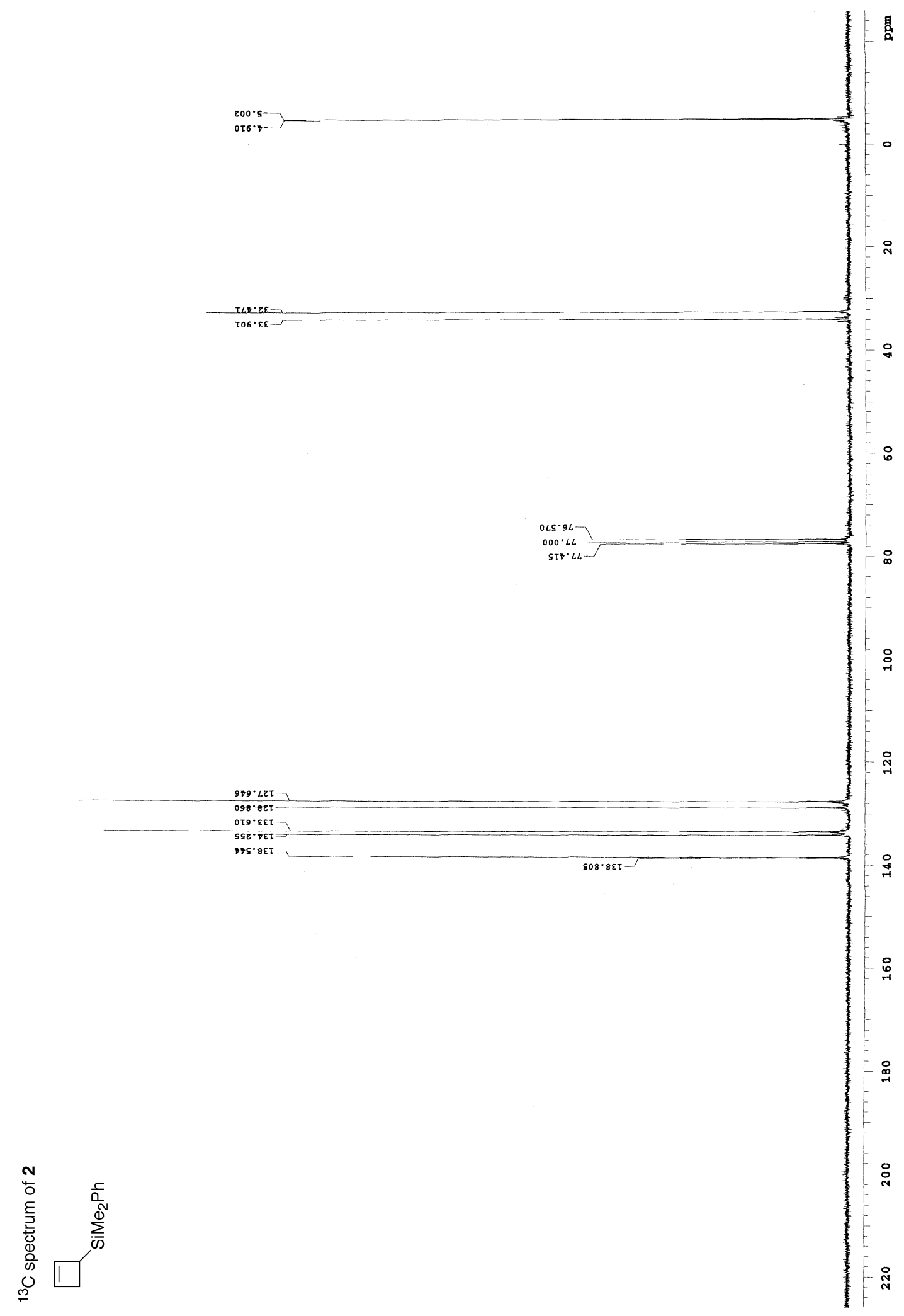




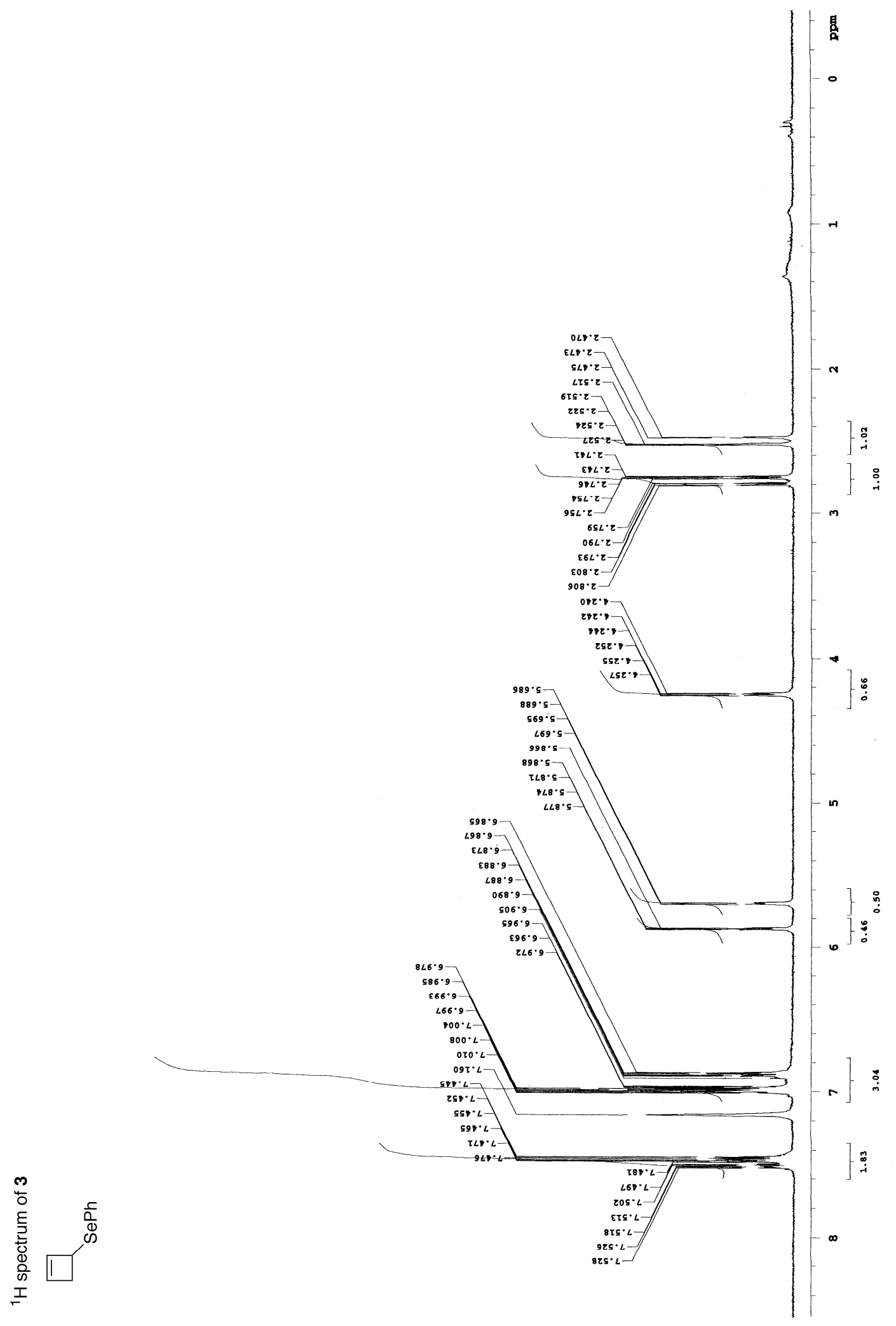




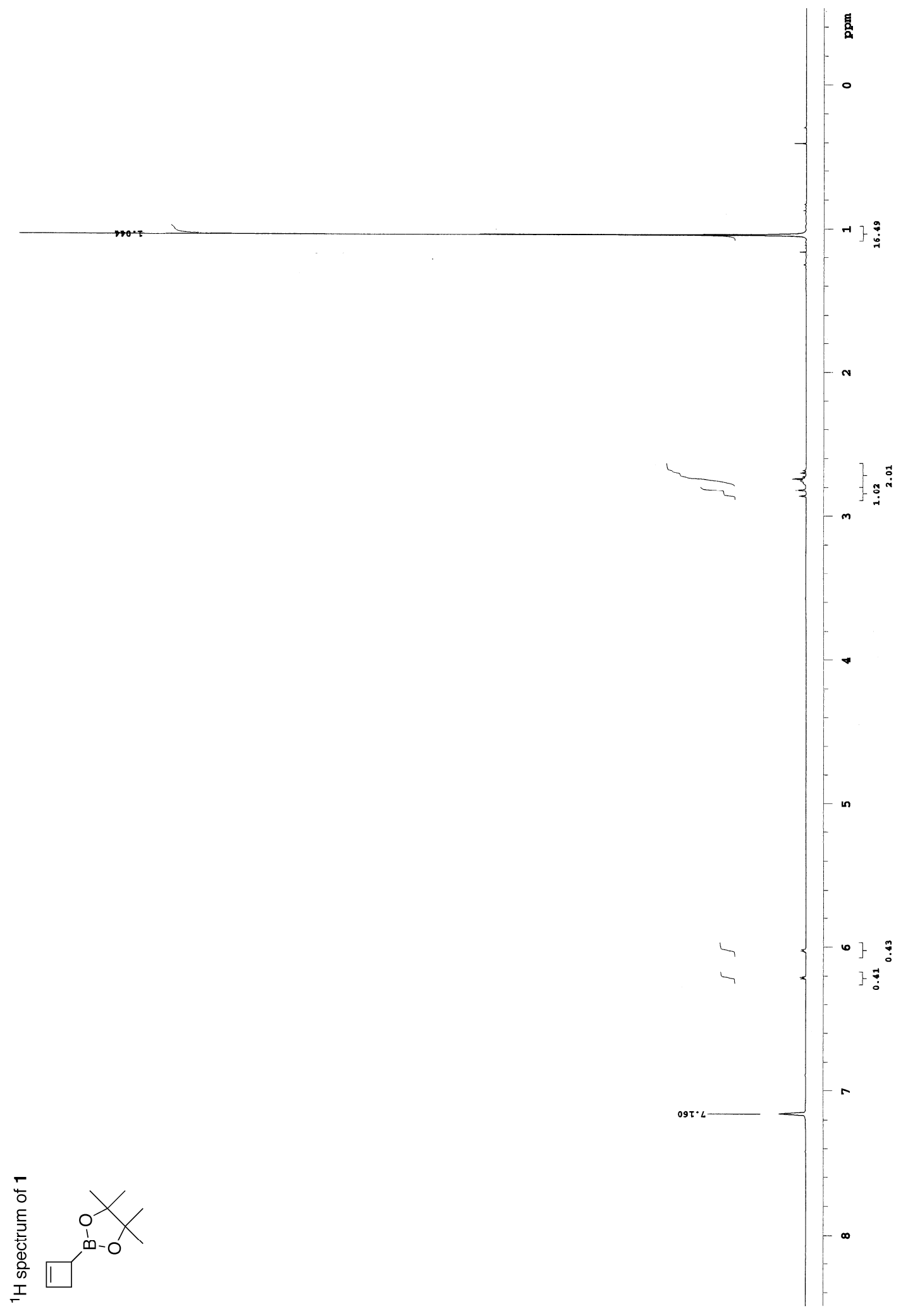




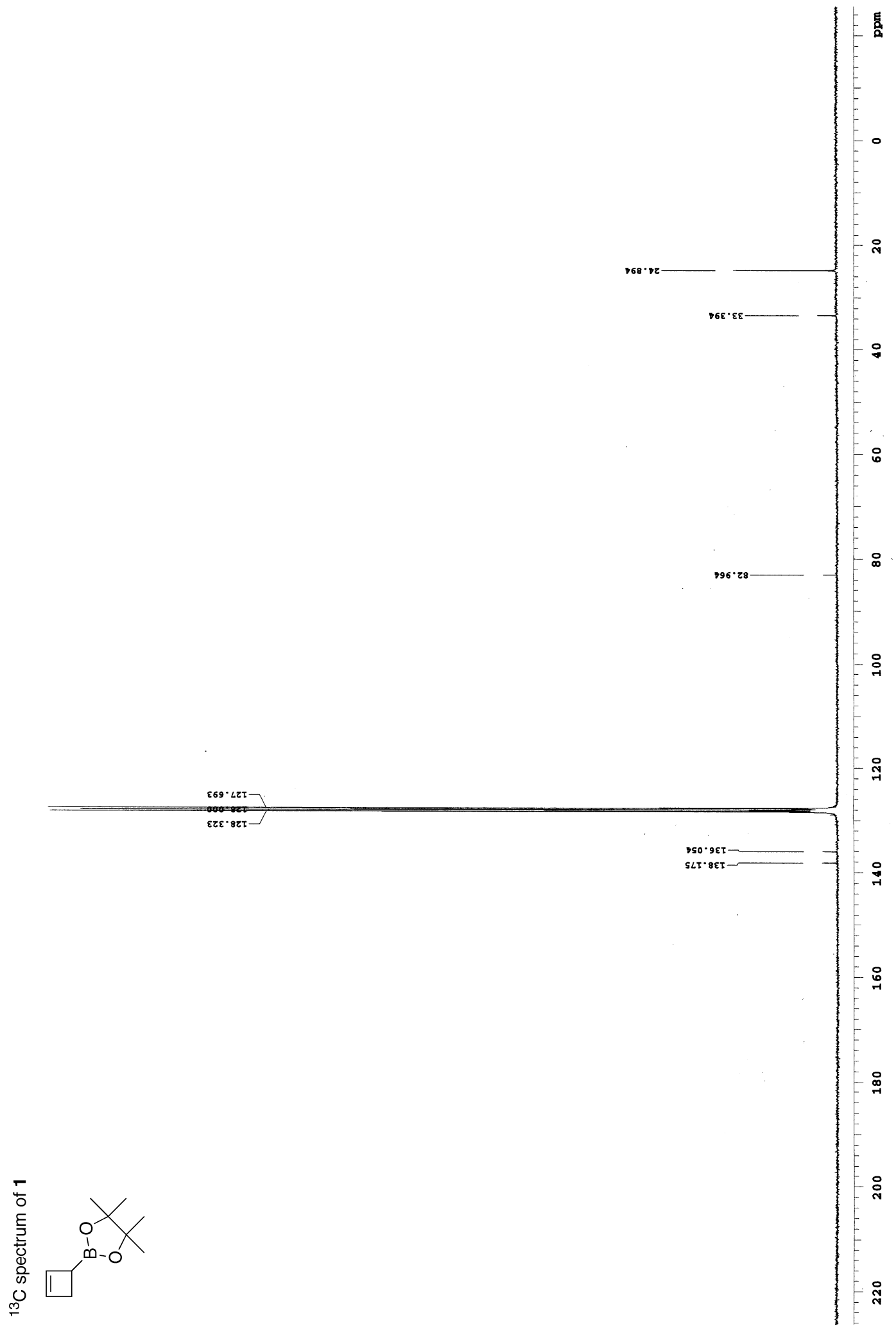




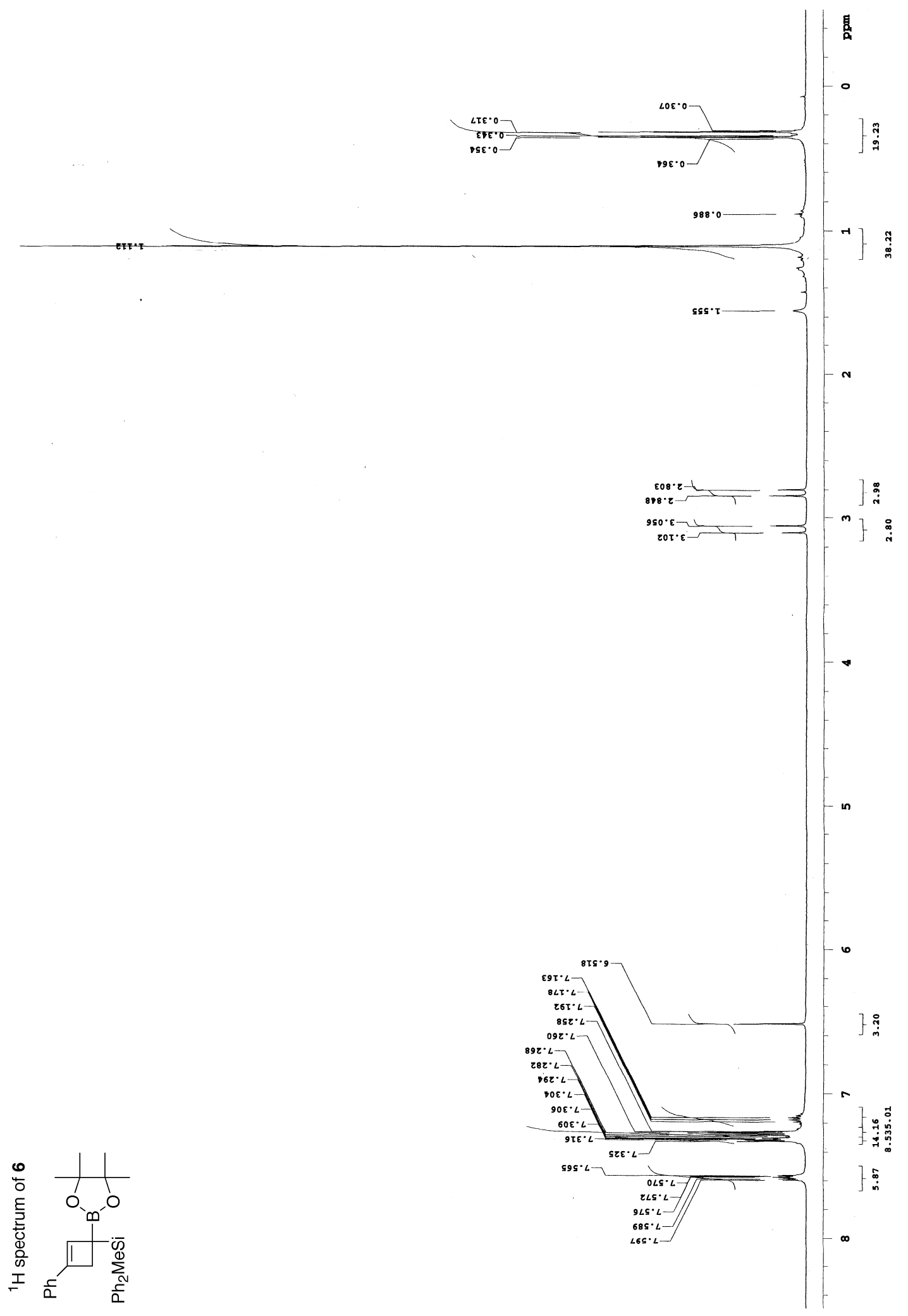




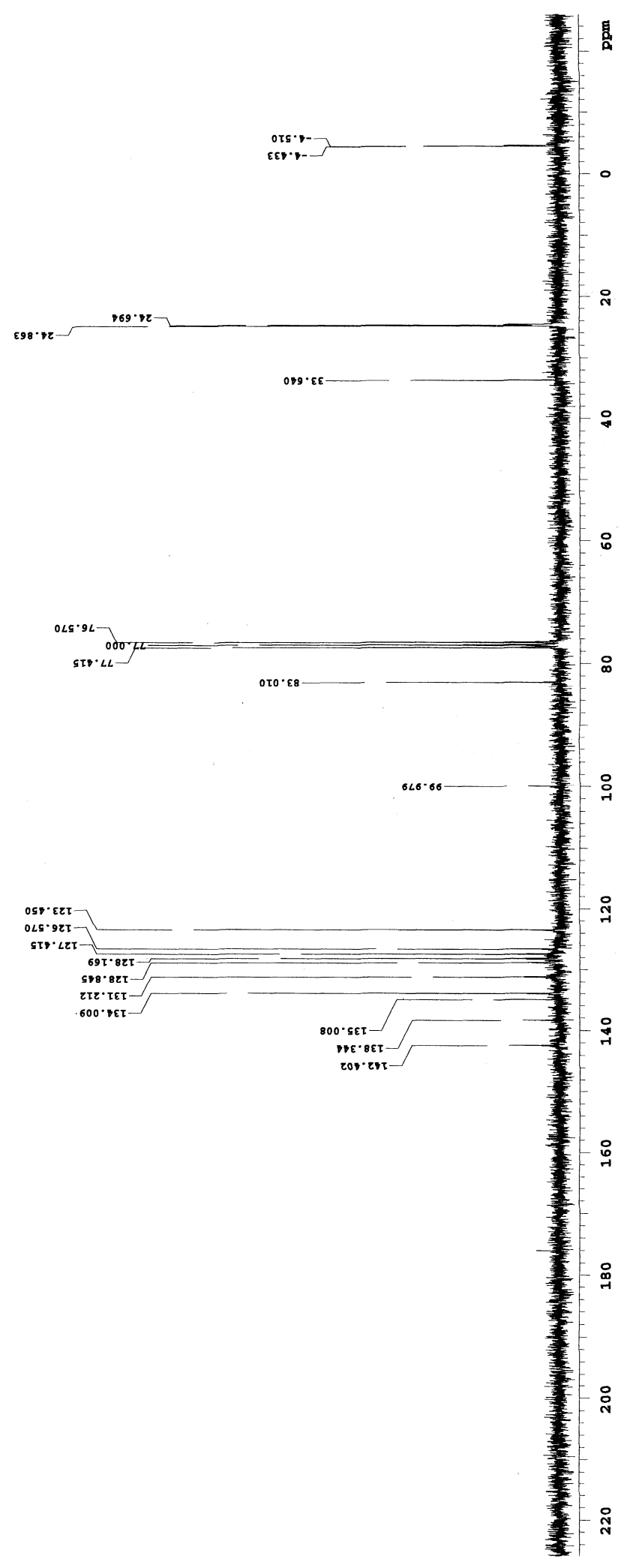




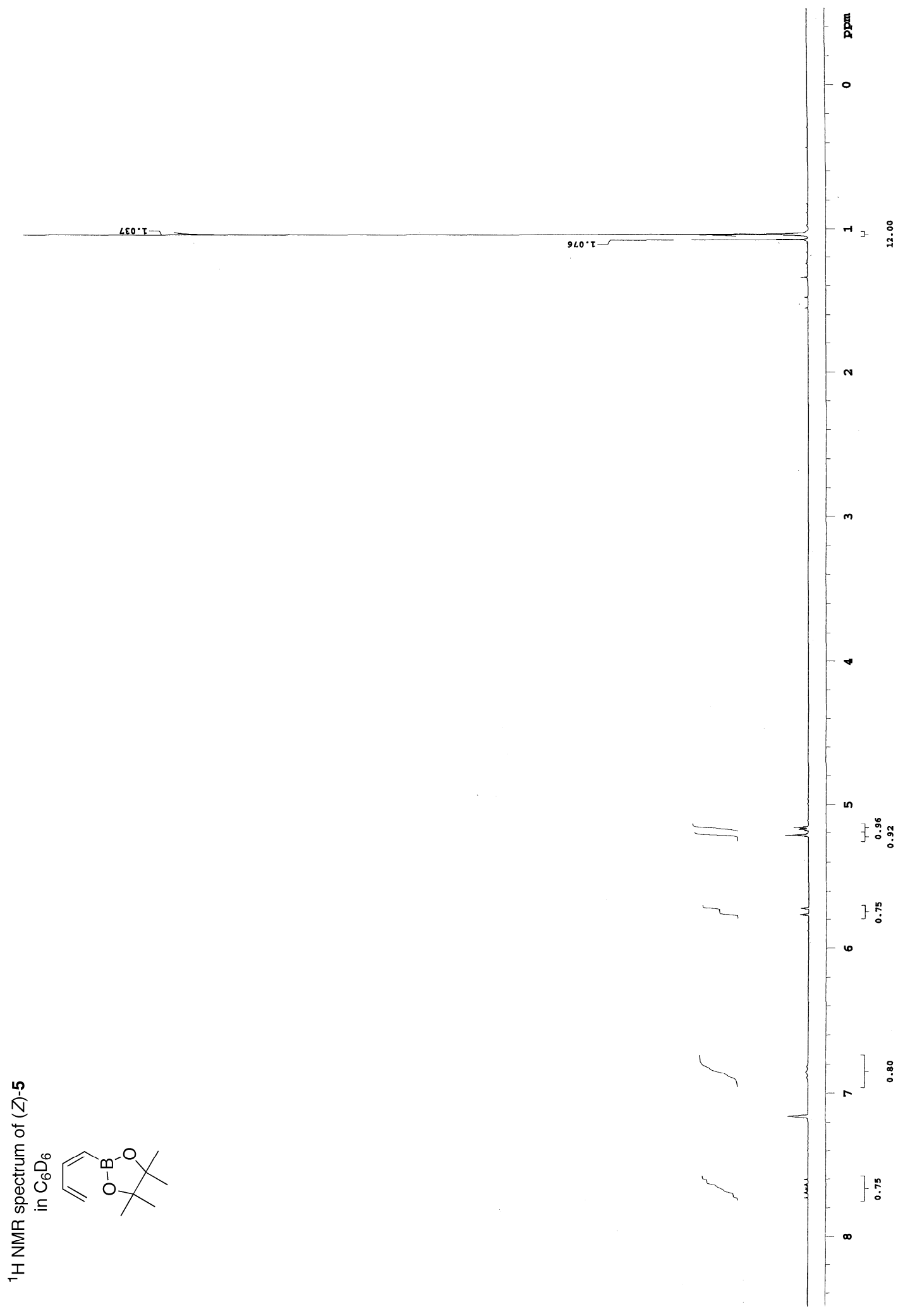




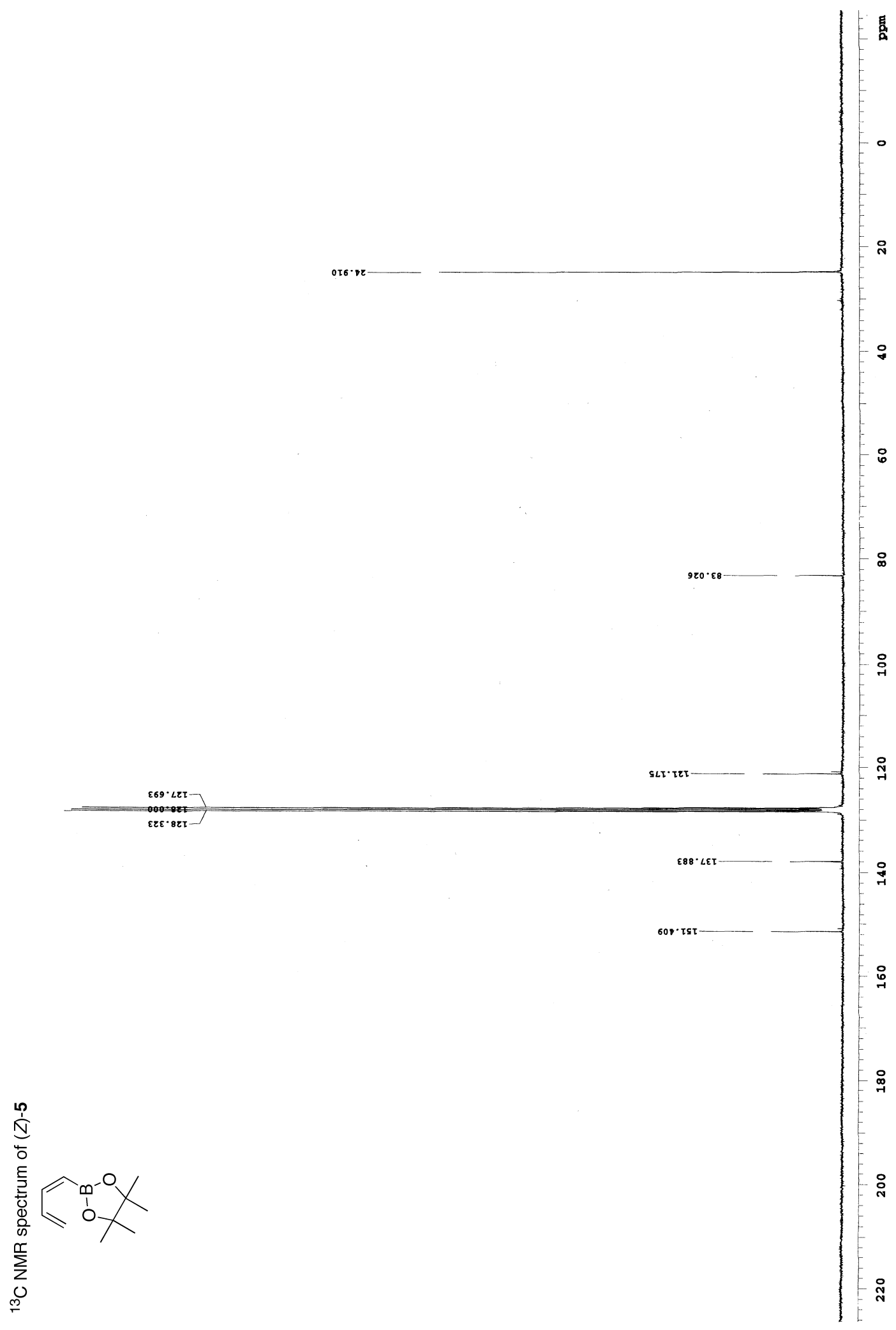




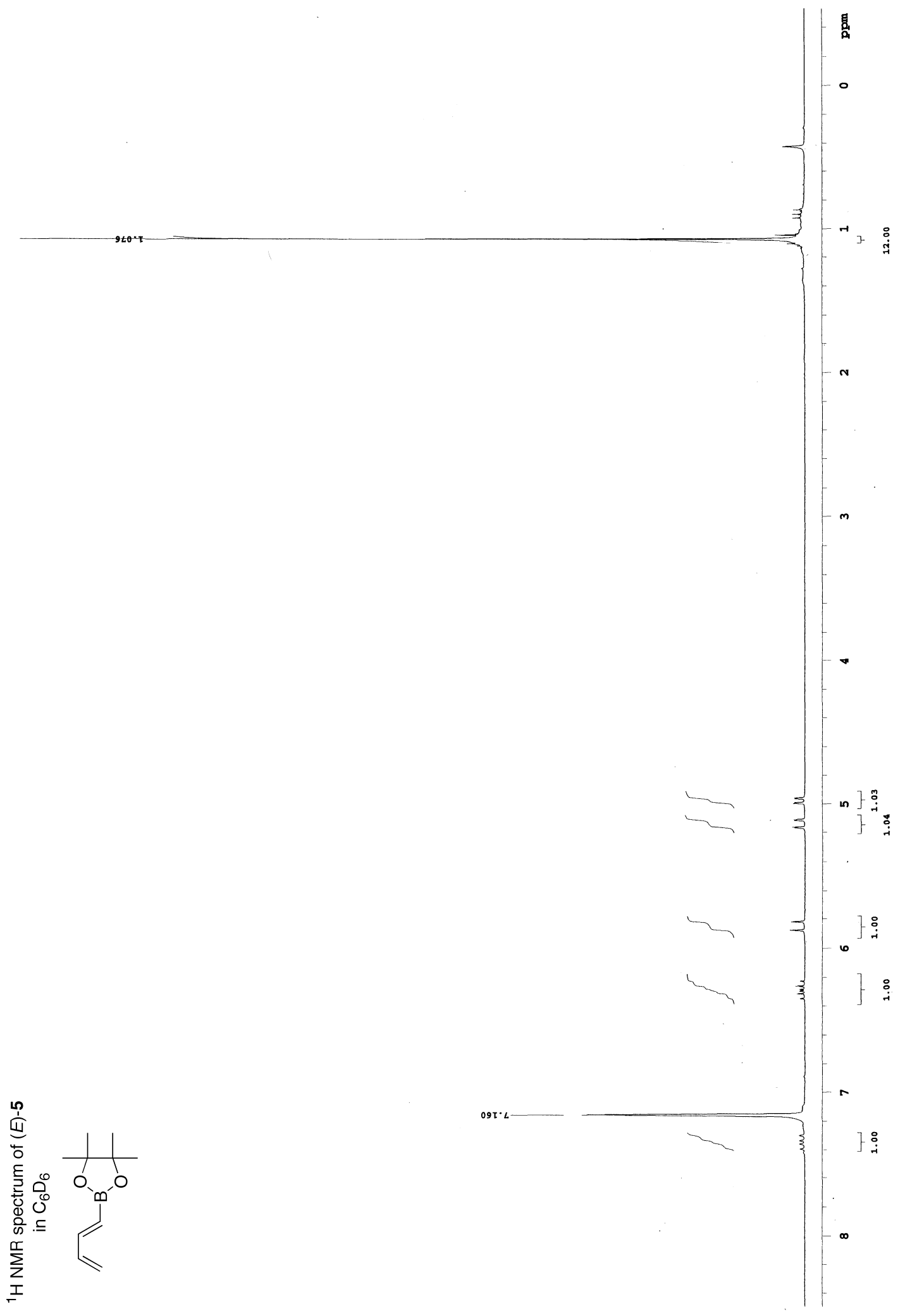




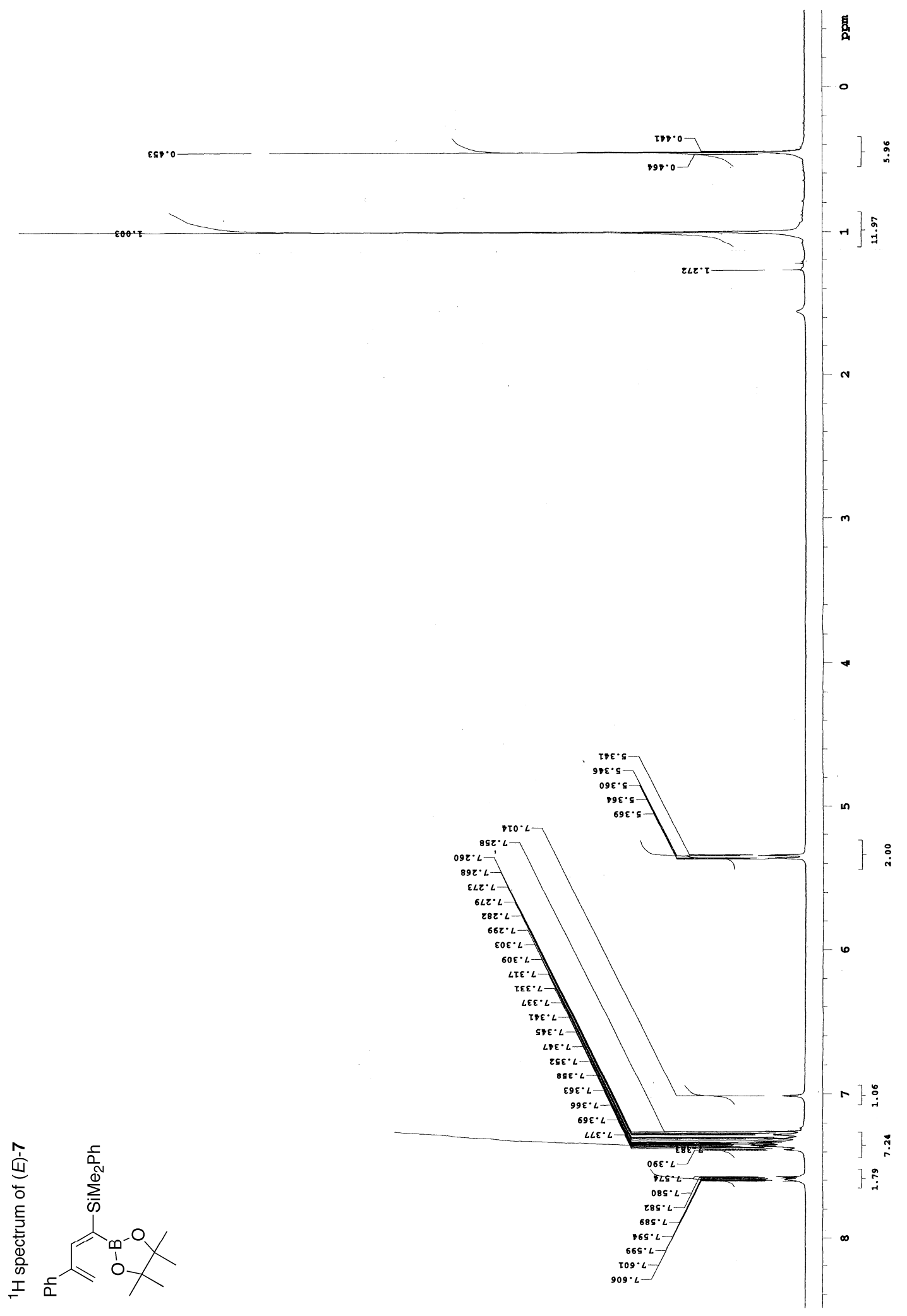




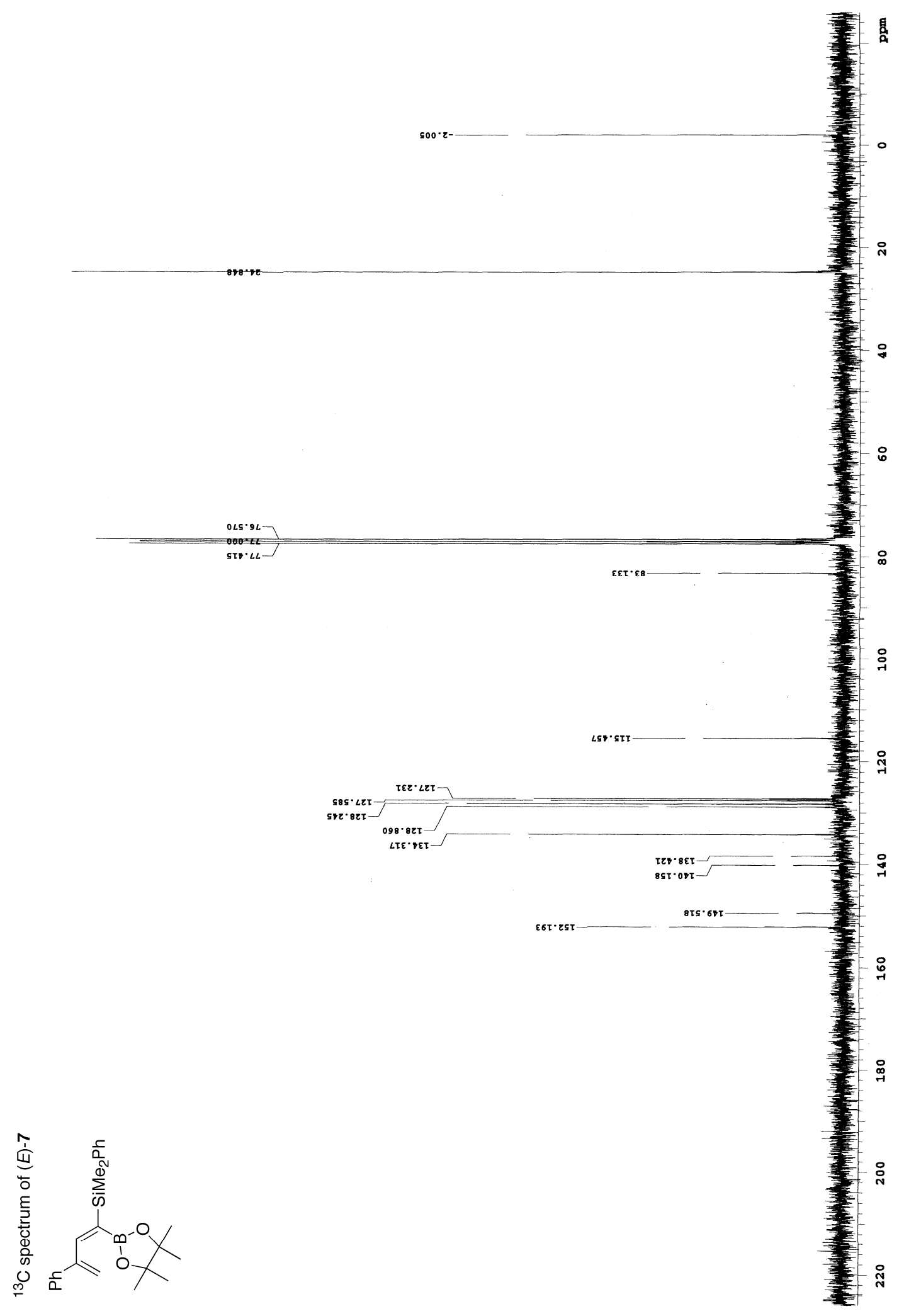




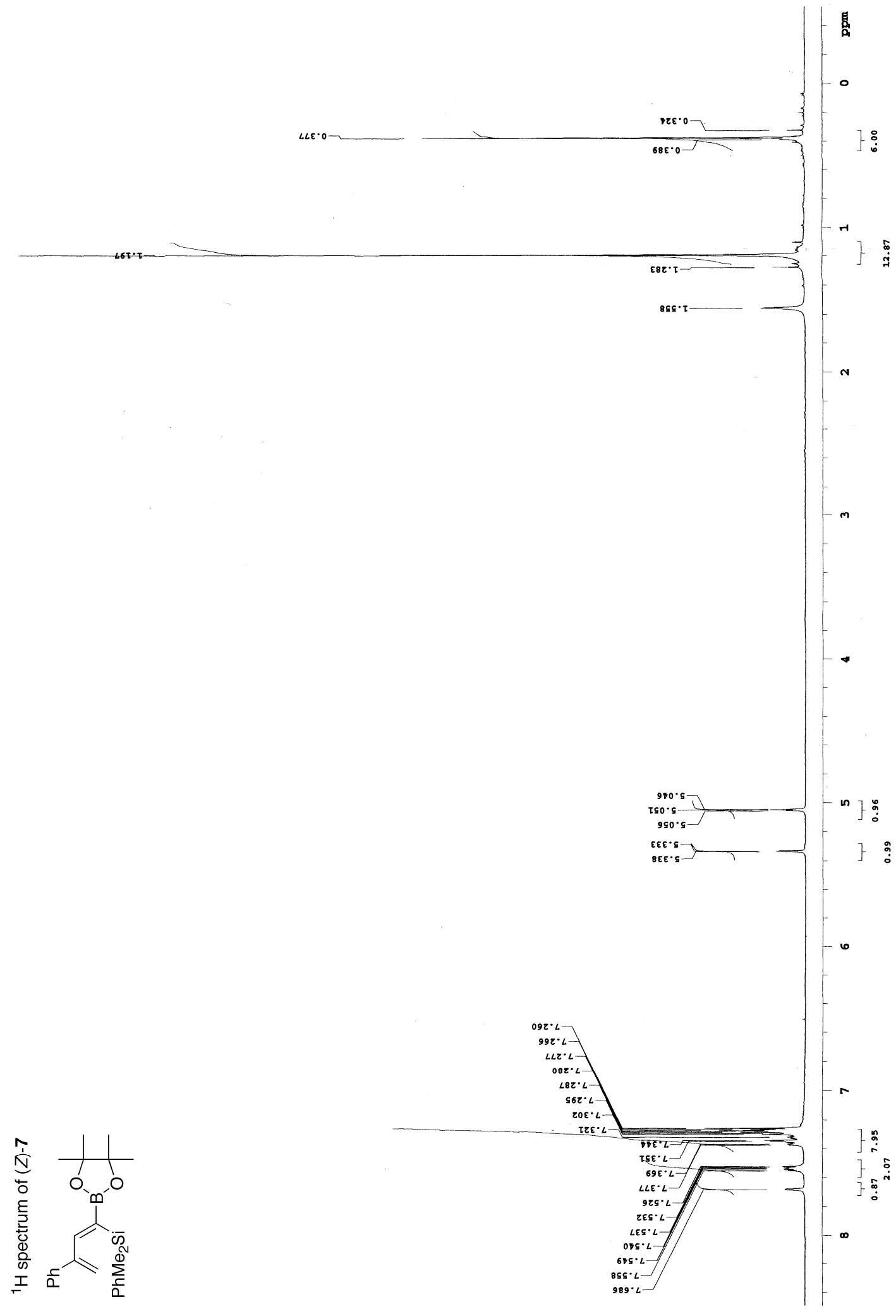




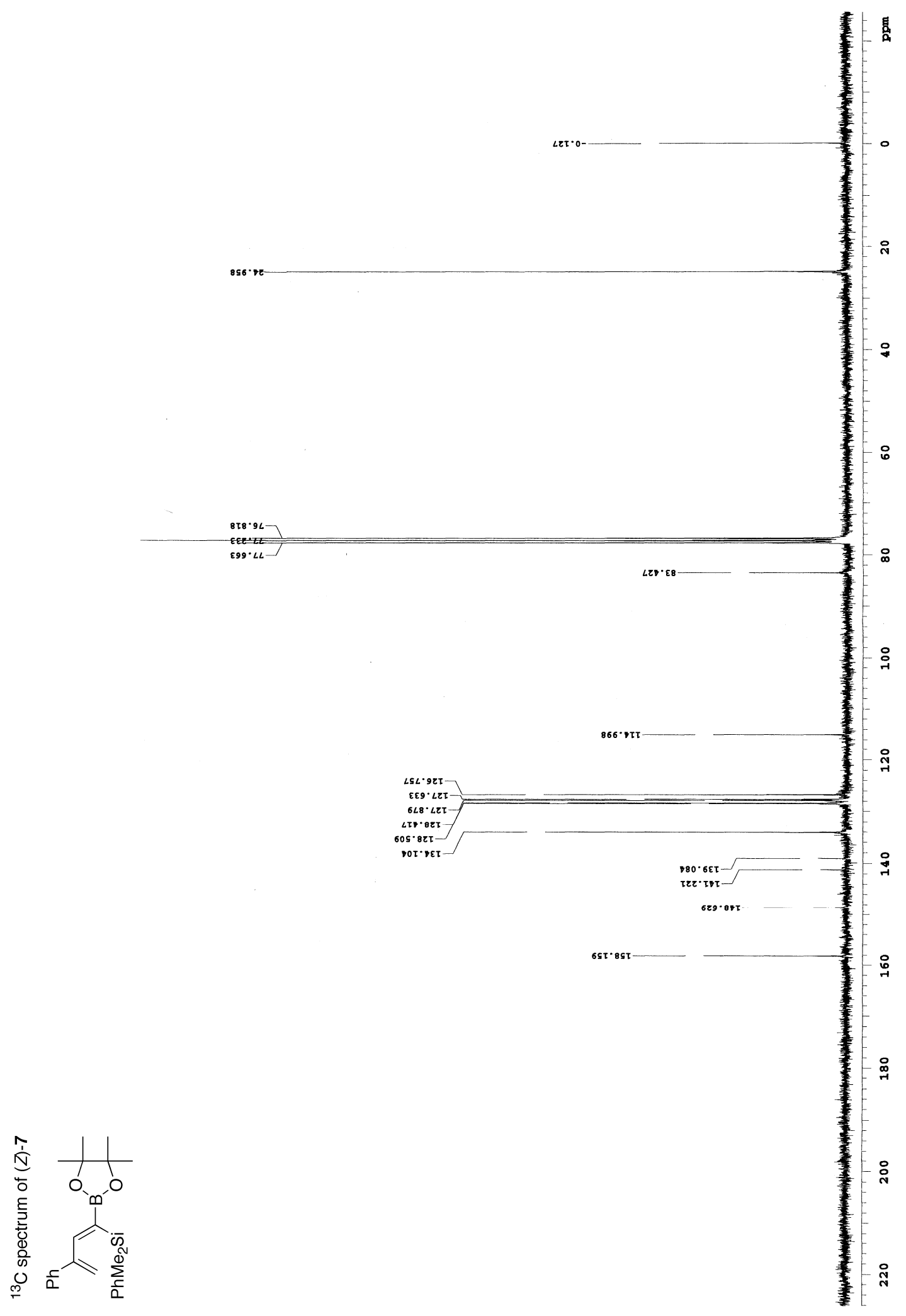

